\title{
Reversible Switching of Organic Diradical Character via Iron-Based Spin-crossover
}

\author{
Airi Kawamura ${ }^{\ddagger 1}$, Jiaze Xie ${ }^{\ddagger 1}$, Jan-Niklas Boyn ${ }^{1}$, Kate A. Jesse ${ }^{1}$, Andrew J. McNeece ${ }^{1}$, Ethan A. Hill1 ${ }^{1}$ \\ Kelsey A. Collins' ${ }^{2}$, Juan A. Valdez-Moreira ${ }^{3}$, Alexander S. Filatov ${ }^{1}$, Josh W. Kurutz ${ }^{1}$, David A. Maz- \\ ziotti, $^{1}$ and John S. Anderson ${ }^{*}$ \\ ${ }^{\dagger}$ These authors contributed equally. ${ }^{1}$ Department of Chemistry, The University of Chicago, Chicago, IL, USA. ${ }^{2}$ De- \\ partment of Chemistry, Northwestern University, Evanston, IL, USA. ${ }^{3}$ Department of Chemistry, Indiana University, \\ Bloomington, IN, USA. *Email: jsanderson@uchicago.edu
}

\begin{abstract}
Organic diradicals are uncommon species that have been intensely studied for their unique properties and potential applicability in a diverse range of innovative fields. While there is a growing class of stable and well characterized organic diradicals, there has been recent focus on how diradical character can be controlled or modulated with external stimuli. Here we demonstrate that a diiron complex bridged by the doubly oxidized ligand tetrathiafulvalene-2,3,6,7tetrathiolate ( $\mathrm{TTFtt}^{2-}$ ) undergoes a thermally induced Fe-centered spin-crossover which yields significant diradical character on TTFtt ${ }^{2-}$. UV-vis-Near-IR, Mössbauer, NMR, and EPR spectroscopies with magnetometry, crystallography, and ad-

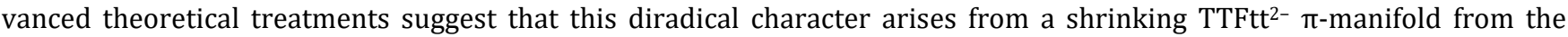
Fe(II)-centered spin-crossover. The $\mathrm{TTFtt}^{2-}$ centered diradical is predicted to have a singlet ground state by theory and variable temperature EPR. This unusual phenomenon demonstrates that inorganic spin transitions can be used to modulate organic

diradical

character.
\end{abstract}

\section{Introduction}

Organic diradicals feature two unpaired electrons which can populate either triplet or open-shell singlet ground states. $^{1}$ These unusual species have attracted attention both for their fundamental properties and for applications in organic semiconductors, non-linear optics, singlet fission, and spintronics. ${ }^{2,3,4,5}$ Significant progress has been made in generating stable species and in understanding their ground states and electronic structures. $6,7, \square, \square$ Tuning or switching diradical character with external stimuli, however, remains challenging. ${ }^{10,11,12,13,14,15}$ This advance is crucial for responsive properties as would be required in spintronics or optical applications.

Spin-crossover is a well-known phenomenon in inorganic chemistry, particularly in six-coordinate Fe(II) complexes. ${ }^{16,17,18}$ The ability to switch between high- $(S=2)$ and low-spin $(S=0)$ states with light (i.e. the LIESST effect) $19,20,21$ or heat makes spin-crossover complexes attractive targets for applications in memory, displays, sensors, and, most relevantly, switches. ${ }^{22}$ Combining spin-crossover phenomena with appropriate organic ligands is therefore a compelling strategy for generating switchable diradicals. Merging radical containing ligands with transition metal centers has been an area of active study. ${ }^{23,24,25}$ While several metal containing organic diradicals have been reported in recent years, these examples are largely limited to transient intermediates or pairs of monoradical ligands. ${ }^{26,27,28,29,30,31}$ The viability of spin-crossover modulated diradical character remains untested.

In targeting radical ligands, the archetypal organic electronic material tetrathiafulvalene (TTF) and its derivatives are attractive targets due their redox-activity and electronic properties. ${ }^{32,33}$ Recently, we have synthesized multiple tetrathiafulvalene-2,3,6,7-tetrathiolate $\left(\mathrm{TTFtt}^{\mathrm{n}-} ; \mathrm{n}=4,3\right.$, and 2) complexes. ${ }^{34}$ While TTF-based diradicals have not been previously reported, we hypothesized that a compressed $\pi$ manifold in oxidized TTFtt $^{2-}$ ligands combined with variable $\pi$ interactions between low- and high-spin Fe centers might enable switchable diradical behavior.

Herein, we report the compounds (FeTPA) 2 TTFtt (1) (TPA = tris(2-pyridylmethyl)amine) and $\left[(\mathrm{FeTPA})_{2} \mathrm{TTFtt}\right]\left[\mathrm{BAr}_{4}\right]_{2} \quad$ (2) $\left(\mathrm{BAr}_{4}{ }_{4}=\right.$ tetrakis $[3,5-$
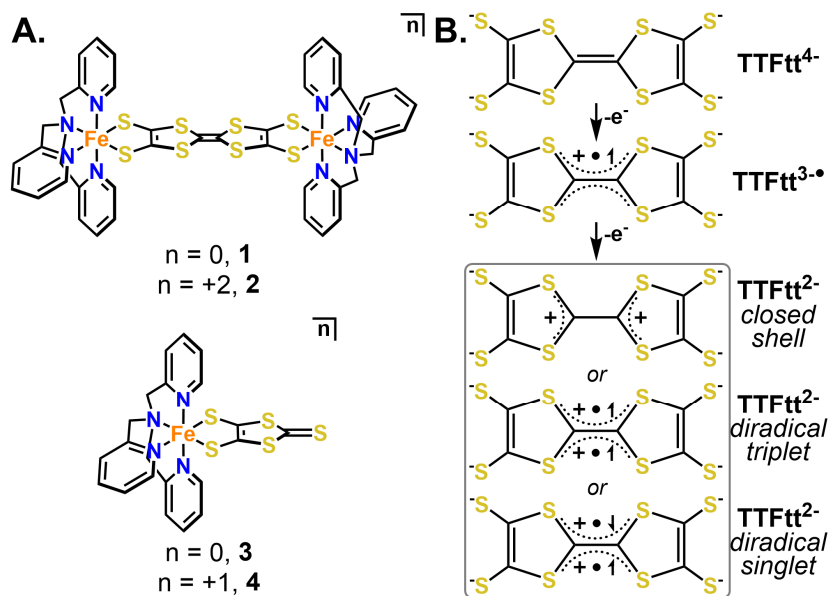

Chart 1. A. Synthesized dinuclear compounds of TTFtt ${ }^{\text {n- }}$ and mononuclear $\mathrm{dmit}^{\mathrm{n}-}$ analogues that serve as half-unit models. B. Possible redox and spin isomers of TTFtt ${ }^{\mathrm{n}-}$ ligands discussed in the text. 


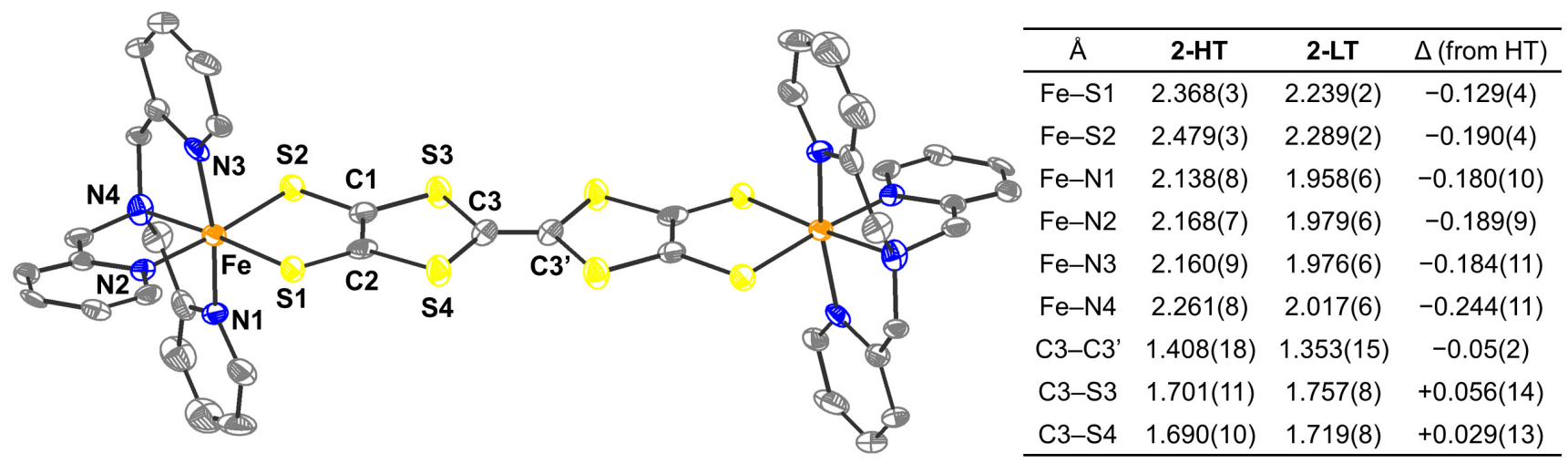

Figure 1. Molecular structure for 2 collected at $100 \mathrm{~K}$ by SXRD (2-LT). H atoms, counterions, and solvent molecules are omitted for clarity and ellipsoids are shown at 50\%. Selected bond length parameters for 2 at $293 \mathrm{~K}$ (2-HT) and $100 \mathrm{~K}$ (2-LT). bis(trifluoromethyl)phenyl]borate) as well as their monomeric analogues Fe(TPA)(dmit) (3) and [Fe(TPA)(dmit)][BAr ${ }^{\mathrm{F}}$ ] (4) (dmit = 1,3-dithiole-2-thione4,5-dithiolate, Chart 1A). Compound 2 represents the first thoroughly characterized example of TTFtt ${ }^{\mathrm{n}-}$ ligands coordinated to $\mathrm{Fe}$ ions. Compound $\mathbf{2}$ exhibits spin-crossover induced switching of TTFtt ${ }^{2-}$-based diradical character and is thus an unusual example showing how inorganic spin transitions can be used to change organic diradical character.

\section{Results and Discussion}

\section{Synthesis and Structural Parameters}

Complex 1 was synthesized via reaction with the deprotected proligand 2,3,6,7-tetrakis(2cyanoethylthio)tetrathiafulvalene $\left(\mathrm{TTFtt}\left(\mathrm{C}_{2} \mathrm{H}_{4} \mathrm{CN}\right)_{4}\right)$ in good yield. Complex 1 was insoluble in all solvents we investigated which precluded detailed characterization but is pure as indicated by combustion analysis and behaves as a suitable synthon for subsequent chemistry. Complex $\mathbf{1}$ can be doubly oxidized with $\left[\mathrm{Cp}_{2} \mathrm{Fe}\right]\left[\mathrm{BAr}^{\mathrm{F}}{ }_{4}\right]$ to form 2 which is more soluble, enabling common solution characterization including ${ }^{1} \mathrm{H}$ NMR and cyclic voltammetry measurements (Figure S1-S2). Oxidation from 1 to $\mathbf{2}$ could be ligandcentered (TTFtt ${ }^{4-} \rightarrow \mathrm{TTFtt}^{2-}$ ), metal-centered (2 Fe(II) $\rightarrow 2$ Fe(III)), or some intermediate case, but the data acquired for 2 supports a TTFtt $^{2-}$ structure arising from ligandcentered oxidation (Chart 1B, see below).

Compound 2 was structurally characterized via singlecrystal X-ray diffraction (SXRD) at 293 K (2-HT; Figure S3) and $100 \mathrm{~K}$ (2-LT; Figure 1). In both structures TTFtt ${ }^{2-}$ is bridged between two TPA-capped Fe centers with two outer-sphere $\mathrm{BAr}^{\mathrm{F}} 4^{-}$counter anions. The most striking difference between these temperatures is markedly longer Fe bond lengths in 2-HT. The Fe- $\mathrm{N}_{\text {pyridine }}$ and $\mathrm{Fe}-\mathrm{N}_{\text {amine }}$ bond lengths in 2-LT are 1.958(6)-1.979(6) and 2.017(6) $\AA$ (Figure 1), respectively. These values are consistent with $\mathrm{Fe}-\mathrm{N}$ bonds in other low-spin complexes with a Fe-TPA moiety. ${ }^{16,17}$ In 2-HT, these bonds are 0.18-0.19 and $0.244(11) \AA$ longer than their counterparts at $100 \mathrm{~K}$, respectively, and are consistent with high-spin Fe-TPA complexes. The shorter Fe bonds at lower temperature indicate that $\mathbf{2}$ exhibits a temperature dependent spincrossover as observed in related compounds. ${ }^{16,21}$
In addition to the geometric changes at $\mathrm{Fe}$, the central $\mathrm{C} 3-\mathrm{C} 3$ ' bond and the central $\mathrm{C}-\mathrm{S}$ bonds (C3-S3 and C3-S4) in the TTF core of $\mathbf{2}$ show temperature dependent differences of $\sim 0.04 \AA$ (Figure 1) suggesting some electronic structure change on this ligand as well. These bonds are markers for the oxidation state of $\mathrm{TTFtt}^{\mathrm{n}}$, where similar increases in the $\mathrm{C} 3-\mathrm{C} 3^{\prime}$ bond length and decreases in the C3-S3/4 bond lengths of $\sim 0.02 \AA$ are correlated to the change from $\mathrm{TTFtt}^{3--}$ to $\mathrm{TTFtt}^{2-}$, potentially suggesting that the geometric changes at Fe and the TTFtt ${ }^{\text {n- }}$ ligand are due to a charge transfer induced spin transition (Table S1). ${ }^{34,35,36}$ Theory and experiment, however, suggest that these changes instead arise from increased diradical character on TTFtt ${ }^{2-}$ upon cooling from 2-HT to 2-LT leading to differential population of $\mathrm{C}-\mathrm{C}$ and $\mathrm{C}-\mathrm{S} \pi$ orbitals (see below). In sum, the structural data on 2 support an Fe centered spin transition with additional electronic structure changes of the TTFtt ${ }^{2-}$ ligand.

The mononuclear compounds $\mathbf{3}$ and $\mathbf{4}$ were also synthesized as half-unit analogues to $\mathbf{1}$ and $\mathbf{2}$, respectively, using the related $\mathrm{dmit}^{2-}$ ligand in place of $\mathrm{TTFtt}^{4-}$. The structure of 3 was determined by SXRD (Figure S4), and the Fe-N bonds fall within in the range commonly observed for high-spin Fe(II). While the data quality for $\mathbf{4}$ was poor, connectivity could be determined by SXRD (Figure S5) and confirms the assigned structure.

\section{Mössbauer Spectroscopy}

The neutral compound 1 exhibits two Fe signals over the temperature range from $250 \mathrm{~K}$ to $80 \mathrm{~K}$ (Figure S6-S9). Both signals are characteristic of TPA ligated 6-coordinate HS Fe(II) with isomer shifts $(\delta)$ of $0.968(2) \mathrm{mm} / \mathrm{s}$ and $1.084(2) \mathrm{mm} / \mathrm{s}$ and quadrupole splitting $\left(\Delta E_{\mathrm{Q}}\right)$ values of $3.588(5) \mathrm{mm} / \mathrm{s}$ and $2.773(1) \mathrm{mm} / \mathrm{s}$ at $80 \mathrm{~K}$. The percent composition of these two signals varies batch-to-batch, and therefore likely represents different packing morphologies of 1 leading to subtle differences at the Fe sites.

At $250 \mathrm{~K}$, the ${ }^{57} \mathrm{Fe}$ Mössbauer spectrum of solid 2 shows a high-spin Fe(II) species comprising 46(5)\% of the sample with an isomer shift $(\delta)$ of $0.880(9) \mathrm{mm} / \mathrm{s}$ and quadrupole splitting $\left(\Delta E_{\mathrm{Q}}\right)$ of $3.22(2) \mathrm{mm} / \mathrm{s}$ (Figure 2A, green). ${ }^{17,37,38,39}$ The remaining sample has a broad, poorly resolved signal (blue) potentially composed of multiple sites with an $\delta$ of $0.23(2) \mathrm{mm} / \mathrm{s}$ and $\Delta E_{\mathrm{Q}}$ of $0.81(2) \mathrm{mm} / \mathrm{s}$ which we assign as low-spin Fe(II) sites. These parameters are outside of the 
typical ranges for both low-spin Fe(II) $(\delta: 0.36-0.52 \mathrm{~mm} / \mathrm{s}$; $\Delta E_{\mathrm{Q}}: 0.23-0.52 \mathrm{~mm} / \mathrm{s}$ ) and low-spin Fe(III) ( $\delta: 0.20-0.28$ $\mathrm{mm} / \mathrm{s} ; \Delta E_{\mathrm{Q}}: 1.5-1.7 \mathrm{~mm} / \mathrm{s}$ ) reported for TPA ligated 6coordinate complexes, but are similar to other low-spin Fe(II) species. ${ }^{40}$

Upon cooling, the proportion of low-spin Fe(II) in 2 increases while the signal for high-spin Fe(II) decreases as expected for spin-crossover. At $80 \mathrm{~K}$ (Figure 2B), the lowspin species (blue) has an $\delta$ of $0.314(1) \mathrm{mm} / \mathrm{s}$ and $\Delta E_{\mathrm{Q}}$ of $0.490(3) \mathrm{mm} / \mathrm{s}$, and constitutes $74(1) \%$ of the sample, while the high-spin species (green; $\delta: 0.9(1), \Delta E_{\mathrm{Q}}: 2.87(2)$ ) $\mathrm{mm} / \mathrm{s})$ comprises $19(1) \%$. A small amount $(<10 \%)$ of an unidentified $\mathrm{Fe}$ species is present (purple; $\delta$ : $0.39(2)$ $\left.\mathrm{mm} / \mathrm{s}, \Delta E_{\mathrm{Q}}: 1.26(4) \mathrm{mm} / \mathrm{s}\right)$. This site may either correspond to some high-spin Fe(III) impurity or another lowspin Fe(II) species, potentially from different solid state packing of 2. EPR features indicative of Fe(III) are absent in $\mathbf{2}$ (see below) suggesting that this small signal does arise from an Fe(II) site in another form of 2 . This is supported by the fact that spectra of frozen solutions of $\mathbf{2}$ in PEG2000 are better fit to only two sites (Figure S10-S11).

If the ligand bond changes between 2-HT and 2-LT represented reduction from $\mathrm{TTFtt}^{2-}$ to radical $\mathrm{TTFtt}^{3-}$ a concomitant, presumably Fe-based, oxidation must be occurring. In this case one would either expect: (i) two fully lo-
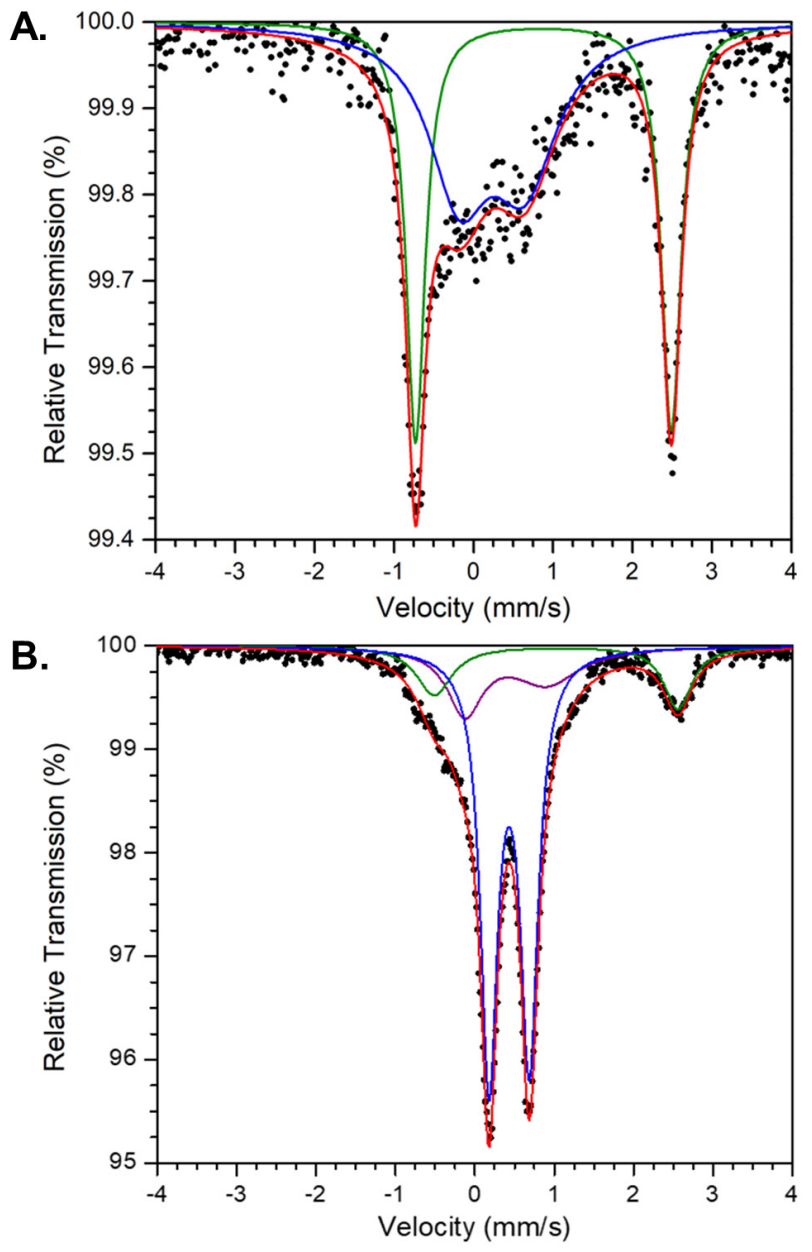

Figure 2. A. ${ }^{57} \mathrm{Fe}$ Mössbauer data for solid 2 collected at $250 \mathrm{~K}$. B. ${ }^{57} \mathrm{Fe}$ Mössbauer data for 2 collected at $80 \mathrm{~K}$. The data is in black, the red line is the overall fit and the blue, green, and purple lines show the fits to the three Fe sites, as outlined in the text. calized, unequal Fe centers (i.e. Fe(II) and Fe(III)) or (ii) two mixed-valent Fe(II/III) centers. The low-temperature Mössbauer data only shows a single major Fe feature eliminating possibility (i). The absence of an inter-valence charge transfer band in the low-temperature electronic spectra of $\mathbf{2}$ (see below) alternatively eliminates possibility (ii). This suggests that $\mathbf{2}$ is predominantly Fe(II) with a $\mathrm{TTFtt}^{2-}$ ligand at all temperatures and any resonance structures invoking Fe(III) with a TTFtt ${ }^{3--}$ ligand are minor contributors. Therefore, the changes in $\mathrm{C}-\mathrm{C}$ and $\mathrm{C}-\mathrm{S}$ bond lengths between 2-HT and 2-LT arise from a different type of change in the electronic structure of TTFtt ${ }^{2-}$.

Complex $\mathbf{3}$ has a straightforward Mössbauer spectrum at both $250 \mathrm{~K}$ and $80 \mathrm{~K}$, which features a single signal characteristic of a HS Fe(II) (Figure S12-S13). The Mössbauer spectrum of 4 has a signal with a $\delta$ of 0.282 to $0.338 \mathrm{~mm} / \mathrm{s}$ and $\Delta E_{\mathrm{Q}}$ of 1.32 to $1.40 \mathrm{~mm} / \mathrm{s}$ between $250 \mathrm{~K}$ and $80 \mathrm{~K}$, respectively (Figure S14-S15). These data indicate that 4 is best thought of as a low-spin Fe(II) center bound to a dmit $^{-}$radical, and corroborate similar low-spin Fe(II) features in 2.

\section{Magnetometry}

The magnetic properties of $\mathbf{1}$ suggest two magnetically isolated high-spin Fe(II) $(S=2)$ centers (Figure S16), but
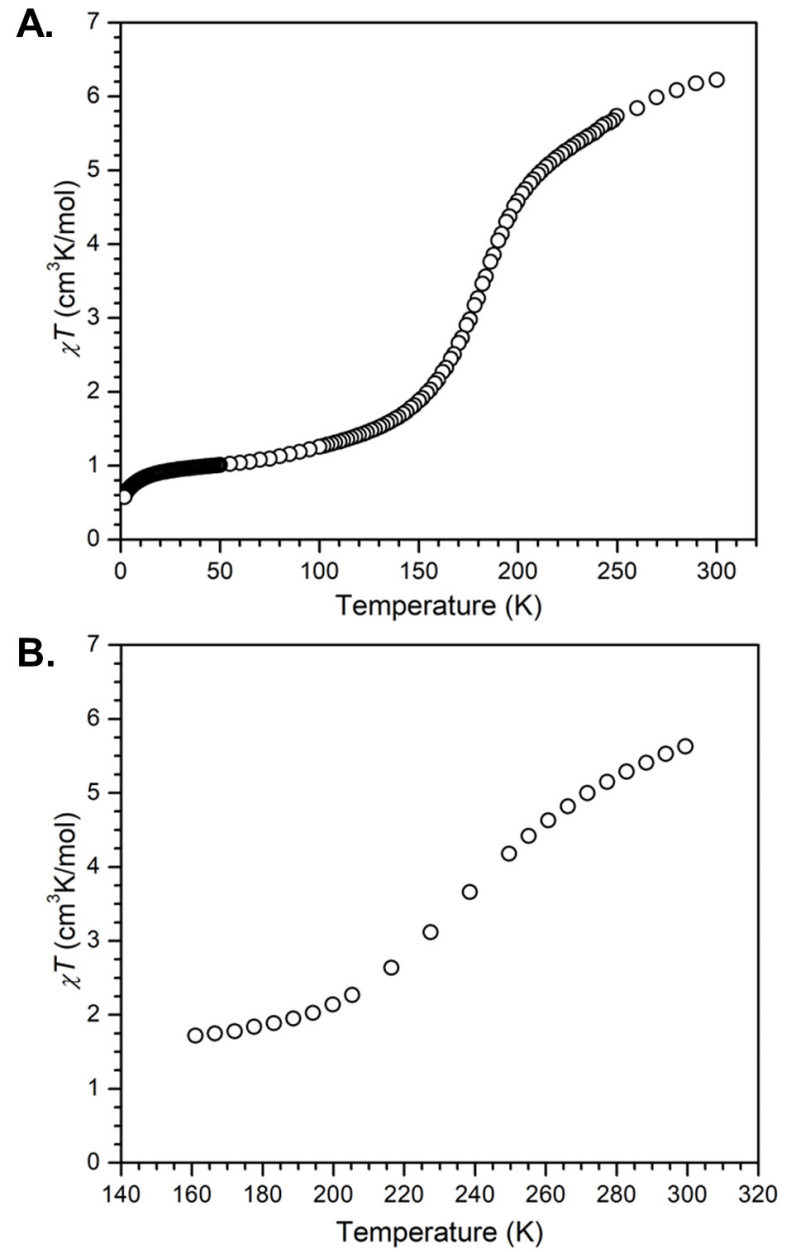

Figure 3. A. Temperature dependent magnetic susceptibility of 2 in the solid state, collected under an applied field of $0.1 \mathrm{~T}$. B. Temperature dependent magnetic susceptibility of $\mathbf{2}$ in 95\% 2-chlorobutane/5\% $\mathrm{C}_{6} \mathrm{D}_{6}$ obtained via the Evans method. 
differ drastically upon oxidation to 2 (Figure $3 \mathrm{~A}$ ). The $\chi T$ of 2 is near the spin-only value of $6 \mathrm{~cm}^{3} \mathrm{~K} / \mathrm{mol}$ at $300 \mathrm{~K}$, but decreases sharply between $\sim 200-150 \mathrm{~K}$. This behavior is completely reversible upon warming (Figure S17). These data indicate two primary regions: high-temperature with high $\chi T$ and low-temperature with low $\chi T$, separated by spin-crossover. From the molecular structures and Mössbauer data in the high- and low-temperature regions, the Fe centers show clear indications of undergoing spincrossover from high- to low-spin, consistent with the observed decrease in $\chi T$. However, a low-temperature $\chi T=0$ would be expected for low-spin Fe(II) $(S=0)$ centers, which is not observed.

One possible explanation is that $\mathbf{2}$ undergoes incomplete spin-crossover as has been previously observed in related systems. ${ }^{21,41}$ Indeed, the magnetic behavior of $\mathbf{2}$ shows high sensitivity to batch effects, which is commonly observed in spin-crossover compounds. ${ }^{42,43}$ However, no sample of 2 shows a $\chi T$ approaching zero. Instead, $\chi T$ is near the values expected for a diradical triplet $\left(S=1, \chi T \sim 1 \mathrm{~cm}^{3} \mathrm{~K} / \mathrm{mol}\right)$ or a diradical system with weak coupling $(2 S=1 / 2, \chi T \sim 0.75$ $\mathrm{cm}^{3} \mathrm{~K} / \mathrm{mol}$ ). Fitting the magnetic data to the Boltzmann equation gives a $\Delta H=1030 \mathrm{~cm}^{-1}$ and $T_{1 / 2}=185 \mathrm{~K}$ (Figure S18)..$^{4}$

Solution phase variable temperature ${ }^{2} \mathrm{H}$ NMR Evans method measurements of $\mathbf{2}$ were performed to determine the $\chi T$ of 2-LT in the absence of possible incomplete spincrossover due to packing effects in the solid state. These data (Figure 3B) show a significant drop upon cooling and a plateau at $\chi T=1.7(1) \mathrm{cm}^{3} \mathrm{~K} / \mathrm{mol}$ at $160 \mathrm{~K}$. Importantly, the electronic spectrum of $\mathbf{2}$ under the same conditions (see below) shows that spin-crossover is complete by $\sim 170 \mathrm{~K}$. This indicates that the $\chi T$ at $160 \mathrm{~K}$ arises from 2LT, which therefore cannot be diamagnetic. The above mentioned Mössbauer data rule out any major resonance contribution from $\mathrm{Fe}(\mathrm{III})$ with a $\mathrm{TTF}^{3--}$ ligand which suggests that the nonzero $\chi T$ across the low temperature region must arise from unpaired spin density on $\mathrm{TTFtt}^{2-}$.
Fitting the solution phase magnetic data to the Boltzmann equation gives $\Delta H=1400 \mathrm{~cm}^{-1}$ and $T_{1 / 2}=260 \mathrm{~K}$ (Figure S19).

The neutral compound $\mathbf{3}$ exhibits magnetic behavior as expected for a HS Fe(II) $(S=2)$ center (Figure S20) and cooling to $1.8 \mathrm{~K}$ does not yield any notable features which would contradict a simple assignment of a HS Fe(II) and a diamagnetic $\mathrm{dmit}^{2-}$ ligand. Monocationic 4 has a $\chi T$ near the expected value for a $S=1 / 2$ species $\left(\chi T_{S O}=0.375\right.$ $\mathrm{cm}^{3} \mathrm{~K} / \mathrm{mol}$; Figure S21). The room temperature solution moment of $\mathbf{4}$ was also measured by Evans method as 2.1(1) $\mu_{\mathrm{B}}\left(0.5 \mathrm{~cm}^{3} \mathrm{~K} / \mathrm{mol}\right.$; Figure S22) and supports the assignment of the species as $S=1 / 2$ overall.

\section{Variable Temperature UV-Vis-NIR Spectroscopy}

The combined SXRD, Mössbauer, and magnetic measurements suggest that the Fe(II) centers in $\mathbf{2}$ undergo spincrossover to a low-spin state upon cooling with an additional change on the TTFtt ${ }^{2-}$ fragment that gives rise to unpaired electrons. The distinct electronic structure dependent changes in the absorption spectra of TTFtt ${ }^{\mathrm{n}}$ - ligands prompted us to collect variable temperature UV-VisNear-IR (UV-Vis-NIR) spectra of 2 (Figure 4). ${ }^{34,45}$ As expected for a species with a diamagnetic TTFtt $^{2-}$ core, 2 shows an intense feature at $1074 \mathrm{~nm}$ in the NIR region. Upon cooling, the $1074 \mathrm{~nm}$ peak decreases in intensity while a broad feature at $1615 \mathrm{~nm}$ and sharper features at 325 and $396 \mathrm{~nm}$ increase. This spectroscopic behavior is fully reversible upon warming. Fitting the normalized intensity of the NIR feature at $1074 \mathrm{~nm}$ to the Boltzmann equation gives a $\Delta H=1500 \mathrm{~cm}^{-1}$ and $T_{1 / 2}=265 \mathrm{~K}$, in good agreement with solution magnetic data (Figure S23). Importantly, the spectra of 3, 4 and [(dppeNi) ${ }_{2} \mathrm{TTFtt}\left[\mathrm{BAr}_{4}{ }_{4}\right]_{2}$, which is well described as a closed shell singlet, do not show similar changes with temperature, supporting fundamentally different properties in $\mathbf{2}$ at low temperature (Figure S24-S26).

Radical TTFtt ${ }^{3-}$ cores have a lower energy NIR feature

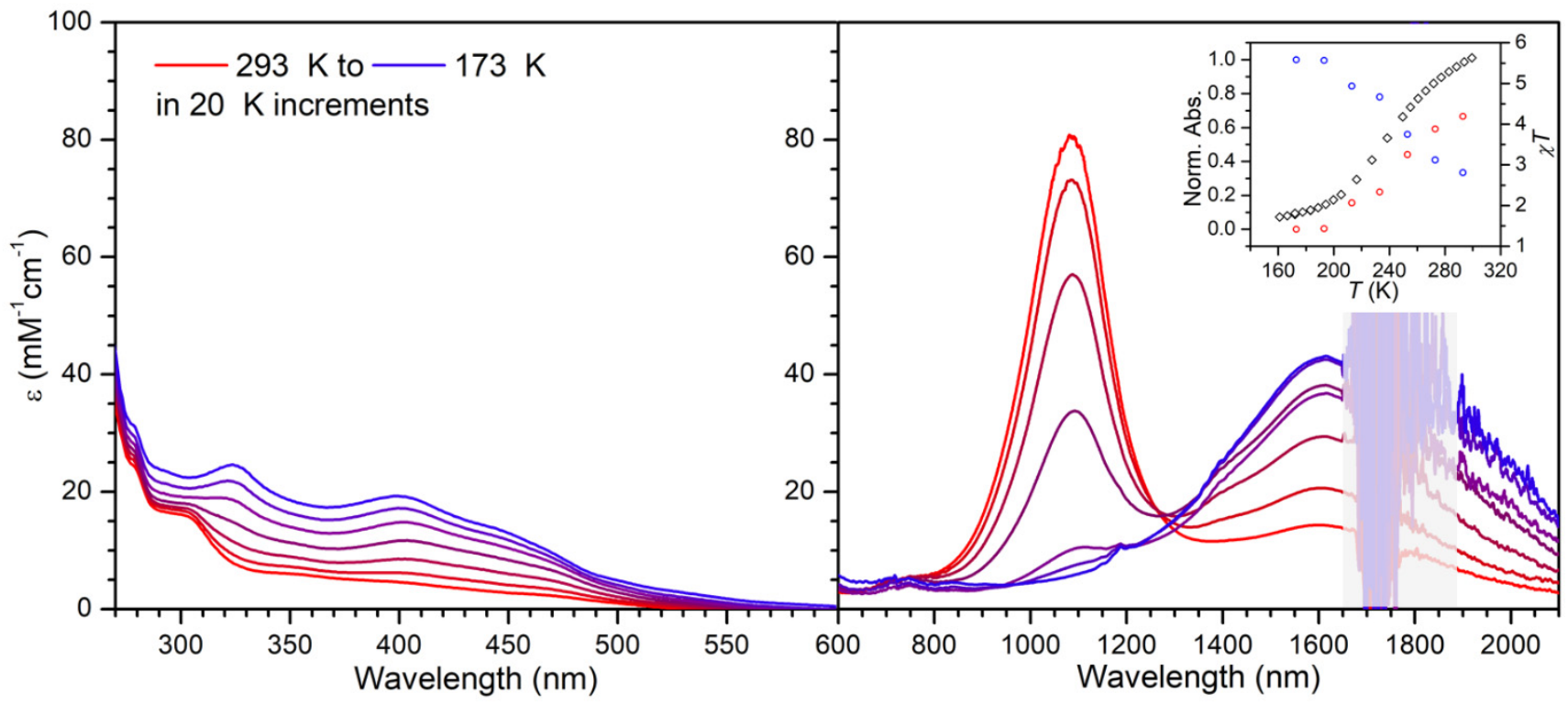

Figure 4. Variable temperature electronic spectra of 2 in the UV-visible (left) and visible-NIR (right) regions collected in 2chlorobutane at $50 \mu \mathrm{M}$. The grey box (right) covers the strong NIR absorbances due to solvent. Inset at right shows the normalized maximum absorbance (left-axis) of the NIR features near $1100 \mathrm{~nm}$ (red) and $1600 \mathrm{~nm}$ (blue) versus temperature, along with the $\chi T$ values (black, right-axis) of 2 obtained via Evans method in $95 \% 2$-chlorobutane $/ 5 \% \mathrm{C}_{6} \mathrm{D}_{6}$. 
A.
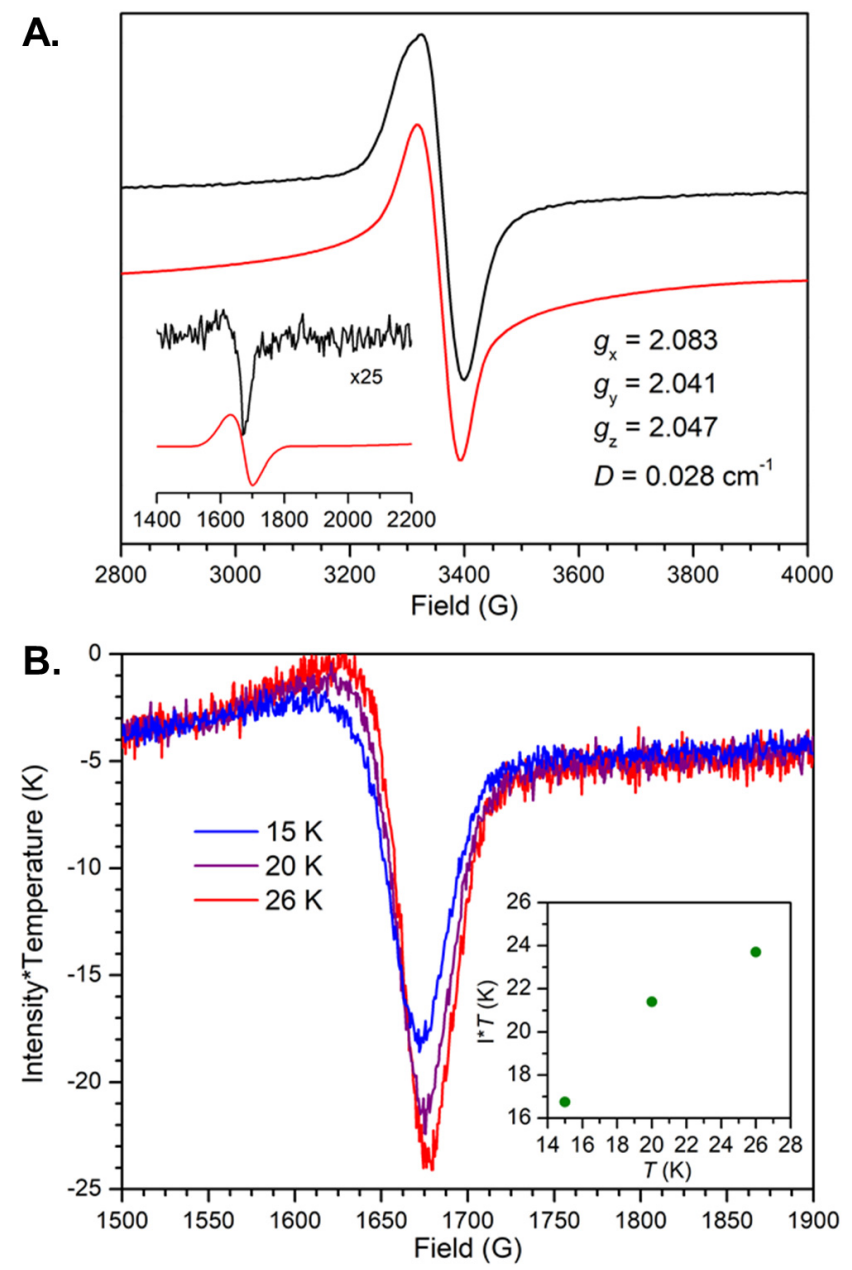

Figure 5. A. X-band EPR spectrum of 2 at $15 \mathrm{~K}$ in DCM at $5 \mathrm{mM}$ with a power of $1.99 \mathrm{~mW}$ and frequency of $9.633 \mathrm{GHz}$. The inset shows the half-field signal centered around $1680 \mathrm{G}$ increased in intensity by a factor of 25 . Red lines indicate simulations with the parameters shown. Experimental data is shown in black. B. Variable temperature change in intensity of the half-field signal of 2 by EPR. Collected in DCM at $5 \mathrm{mM}$. Baselines have been normalized by shifting to overlap. Inset: Peak-to-peak intensity times temperature of the half-field signal versus temperature.

than diamagnetic TTFtt $^{2-}$ cores $^{34,45,46}$ Additionally, the new high energy features ( 325 and $396 \mathrm{~nm}$ ) of 2 at low temperature are similar to those reported for radical TTFtt ${ }^{3-}$. complexes attributed to $\pi \rightarrow \pi^{*}$ transitions. ${ }^{35}$ However, these features in $\mathbf{2}$ at low temperature cannot be due to reduction of the $\mathrm{TTFtt}^{2-}$ ligand to $\mathrm{TTFtt}^{3-}$ as there is no significant oxidation of Fe upon cooling. We therefore used time-dependent density functional theory (TD-DFT) to better understand the observed features in the UV-Vis-NIR spectra.

Ground states for 2-HT ( $S=4)$ and for two potential forms of 2-LT (diradical triplet, $S=1$, with spin density localized on TTFtt $^{2-}$ and closed shell singlet, $S=0$ ) were calculated using the corresponding SXRD structures. Similar calculations were carried out for [(dppeNi) $)_{2} \mathrm{TTFtt}_{[}\left[\mathrm{BAr}_{4}\right]_{2}$ as a comparison and calibration standard (Figure S27). The NIR transitions were well predicted from TD-DFT as TTFtt ${ }^{2-} \pi$-based transitions (Figure
S28-S30). Importantly, the calculated spectrum of the $S=1$ diradical closely matches the low-temperature NIR feature in 2-LT (Figure S31). The prominent NIR transition of 2 at $293 \mathrm{~K}$ also closely matches the calculated features of species with closed-shell forms of TTFtt $^{2-}$, specifically 2-HT (S $=4$, with $\sim 90 \%$ of spin density localized on the FeTPA fragments), the calculated closed-shell $S=0$ state of 2-LT, and the known singlet $\left[(\mathrm{dppeNi})_{2} \mathrm{TTFtt}\right]\left[\mathrm{BArF}_{4}\right]_{2} \cdot{ }^{34}$ This analysis explains the observed UV-vis-NIR features in $\mathbf{2}$ and suggests that the $\mathrm{TTFtt}^{2-}$ ligand gains substantial diradical character upon Fe centered spin-crossover.

The electronic spectrum of $\mathbf{3}$ in DCM was collected at room temperature and features two absorbances at 310 and $498 \mathrm{~nm}$ (Figure S32), assignable to dmit transitions. ${ }^{47,48}$ In the visible region, 4 has a strong peak at 417 $\mathrm{nm}$ with a shoulder near $370 \mathrm{~nm}$ (Figure S32). As this absorbance is between the typical range of a $\mathrm{Fe}(\mathrm{II}) \rightarrow \mathrm{TPA}$ metal-to-ligand charge transfer and the previously reported $\pi \rightarrow \pi^{*}$ transition of the dmit $^{2-}$ ligand, it is not straightforward to assign based on the room temperature spectrum alone. ${ }^{49,62}$ A broad band centered near $1050 \mathrm{~nm}$ is present in $\mathbf{4}$, and notably absent in $\mathbf{3}$. A broad absorbance in this range has been observed to appear when oxidizing a $\mathrm{dmit}^{2-}$ containing complex to the radical dmit"- species. $^{.50,51}$ Upon cooling to $193 \mathrm{~K}$, the high energy visible features increase in intensity, although to a lesser degree than was observed in 2 .

\section{EPR Spectroscopy}

The above data on 2 suggests that Fe spin-crossover mediates increased diradical character on the $\mathrm{TTFtt}^{2-}$ ligand so we employed EPR spectroscopy to directly probe this proposed diradical character. The EPR spectrum of $\mathbf{2}$ at $15 \mathrm{~K}$ in DCM displays an intense signal near $g=2$, consistent with a $\left|\Delta m_{s}\right|=1$ transition of an organic $S=1 / 2$ or $S=$ 1 species (Figure $5 \mathrm{~A}$ ). Importantly, a much lower intensity feature around $g=4$ is observed, characteristic of the $\left|\Delta m_{s}\right|$ $=2$ feature of organic diradical species. ${ }^{1,6,10}$ Using the relative intensity of the $\left|\Delta m_{s}\right|=2$ and 1 signals, the distance between the radicals can be estimated. ${ }^{1}$ In 2 this value is around 3.3-4.6 $\AA$ which is similar to the centroid-tocentroid distance between the two 5 -membered rings in TTF $(\sim 4.1 \AA)$. The observed signals for 2 are distinct from possible impurities, ${ }^{42}$ are reproducible over several samples (Figure S33-S34), and are reasonably well simulated as a diradical (Figure $5 \mathrm{~A}$, red line). The intensity (I) of the $\left|\Delta m_{s}\right|=2$ signal of 2 between 26-15 K implies a singlet (i.e. antiferromagnetically coupled diradical) ground-state wherein a decrease in IT upon cooling is expected as the EPR active triplet state is depopulated in favor of the EPR silent singlet state (Figure $5 \mathrm{~B}$ ). ${ }^{1}$ Overall, these data support that 2-LT has significant diradical character. The EPR spectrum of 4 shows a nearly isotropic signal near $g=2$, confirming the organic radical character of this species and supporting a low-spin assignment for the Fe center (Figure S35).

\section{Computational Results}

Advanced theoretical techniques were utilized to predict and understand the electronic character of the $\mathrm{TTFtt}^{2-}$ ligand. Calculations were performed on 2-LT using experi- 
A.

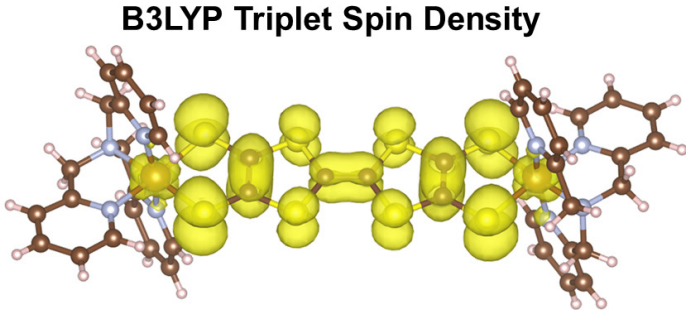

B.

\begin{tabular}{|c|c|c|c|}
\hline \multicolumn{4}{|c|}{ DFT Spin Density } \\
\hline & $\rho \mathrm{Fe}_{1}$ & $\rho \mathrm{Fe}_{2}$ & $\rho$ TTFtt \\
\hline HT & 3.69 & 3.87 & 0.25 \\
\hline LT & 0.13 & 0.13 & 1.73 \\
\hline
\end{tabular}

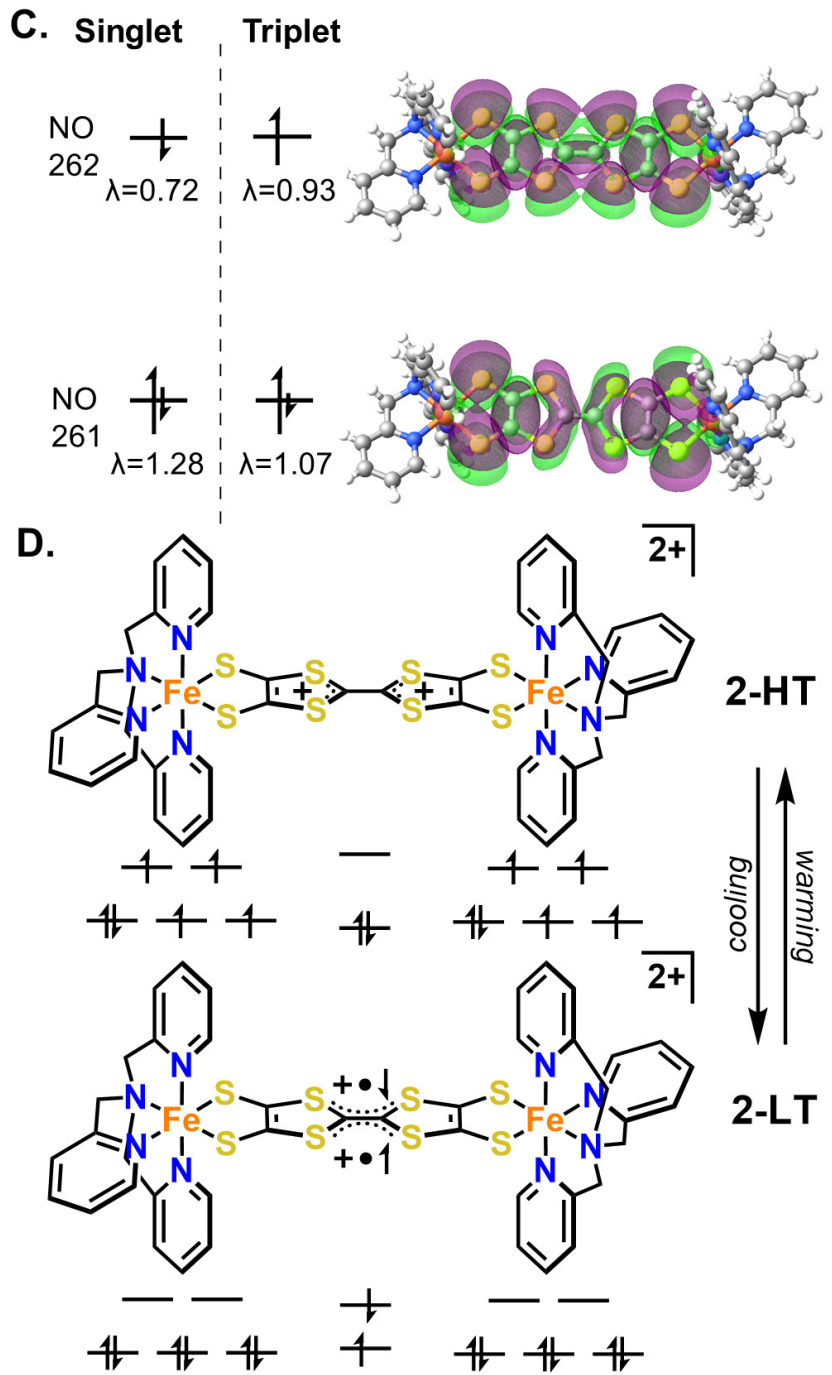

Figure 6. A. Spin density obtained for the triplet state of 2-LT in DFT with the B3LYP functional and a $6-311 \mathrm{G}^{*}$ basis set as implemented in g16/a.01. B. Spin density obtained for the triplet state of 2-LT and 2-HT in DFT with the B3LYP functional and a 6-311G* basis set as implemented in g16/a.01. The values for "Fe" includes all density on the FeTPA fragment. C. Partially occupied frontier NOs and their corresponding NON of 2-LT from a diradical state $[18,20]$ V2RDM calculation with a 6-31G basis set. D. Dominant resonance structures for 2-HT and 2-LT with qualitative frontier orbital diagrams.

mental geometries obtained via SXRD. DFT calculations with the B3LYP functional ${ }^{52}$ and a $6-311 \mathrm{G}^{*}$ basis set ${ }^{53}$ as implemented in g16/a.01, ${ }^{54}$ yielded a triplet ground state with a singlet-triplet gap of $\Delta \mathrm{E}(\mathrm{S}-\mathrm{T})=409 \mathrm{~cm}^{-1}$. The obtained spin density of the triplet state (Figure 6A,B) shows the vast majority of the unpaired electron density is localized on the linker with $\rho \mathrm{Fe}=0.135$ on each FeTPA fragment, and $\rho$ TTFtt $=1.730$.

Given the fact that DFT is not expected to accurately describe the complex electronic structure of open-shell singlet states we turned towards advanced theoretical techniques to validate the B3LYP results. Variational 2-electron reduced density matrix (V2RDM) calculations ${ }^{55,56,57}$ were run in Maple 2019 Quantum Chemistry Package (QCP) ${ }^{58}$ with a $[18,20]$ active space and a 6-31G basis set, covering the entire spin manifold of singlet, triplet, quintet, septet, and nonet states. The results predict the ground state of $\mathbf{2 -}$ LT to be a singlet $\left(\Delta \mathrm{E}(\mathrm{S}-\mathrm{T})=-373 \mathrm{~cm}^{-1}\right)$ with strongly correlated, diradical character and frontier natural occupation numbers (NON) of $\lambda_{261}=1.28$ and $\lambda_{262}=0.72$ (Figure 6C, Table S3). Inspection of the frontier NOs reveals the diradical to be localized almost exclusively on the TTF $\mathrm{tt}^{2-}$ linker with negligible involvement of the Fe d-orbitals, in good agreement with the triplet result from DFT. Hartree Fock MO coefficients reveal similar distributions. Further calculations were performed to verify that these results were not an artifact of the choice of basis set or orbitals (see SI, Table S2).

Additional calculations were performed on the $S=4$ ground state of 2-HT (Figure S36). In 2-HT weakening of the interaction between the Fe d-orbitals and the ligand orbitals raises the metal-based orbitals to yield eight singly occupied, Fe-based, frontier NOs. These results show a significant reduction in diradical character in the organic TTFtt $^{2-}$ linker in 2-HT, as compared to 2-LT. The unpaired electron density previously localized on the TTFtt ${ }^{2-} \pi$ system in 2-LT $(\rho$ TTFtt $=1.730)$ is instead in Fe-based NOs in 2-HT, with $\rho$ TTFtt $=0.25$.

The computational analysis clearly supports the assignment of a TTFtt ${ }^{2-}$-based, strongly correlated diradical with open shell singlet and triplet states close in energy in 2-LT. The temperature driven Fe-based spin transition gives rise to enhanced diradical character on the TTFtt ${ }^{2-}$ core, that is an increase in the occupancy of the higher-lying NO262 at the expense of decreased occupancy in the lower-lying N0261. The DFT calculations suggest that this arises from the shrinking of the energy gap between the NO261 and N0262 in 2-LT (19.59 kcal/mol), as compared to the analogous orbitals (NO254 and NO 268, respectively) in 2-HT (26.01 kcal/mol).

The spin-crossover from 2-HT to 2-LT gives rise to enhanced diradical character on the TTFtt ${ }^{2-}$ core. This spin transfer moves electron density from a $\mathrm{C}-\mathrm{C}$ bonding and C-S antibonding orbital (NO 262 in 2-LT, NO 268 in 2-HT) to a C-C antibonding and C-S bonding orbital (NO 261 in 2-LT, NO 254 in 2-HT, Figure 6D). These changes in orbital populations correlate with the observed variations in bond lengths (Figure 1) and suggest that there should be bond length changes of similar trend, but of potentially larger magnitude when $\mathrm{TTFtt}^{2-}$ undergoes a spin transition as compared to an electron transfer. This is indeed the case when comparing the larger bond length changes of $\mathbf{2}$ ver- 
sus those between $\left[(\mathrm{dppeNi})_{2} \mathrm{TTFtt}_{[}\left[\mathrm{BAr}_{4}\right]_{2}\right.$ and [(dppeNi) 2 TTFtt] [BArF $\left.{ }_{4}\right]$ (Table S1). ${ }^{34}$ Finally, as illustrated above with TD-DFT calculations, this orbital picture also supports the observed spectroscopic changes in 2 . Taken together, all the computational analysis is consistent with the experimental data supporting that an Fe-based spin transition shrinks the gap between TTFtt ${ }^{2-}$-based orbitals thereby increasing organic diradical character.

\section{Conclusions}

We have synthesized and characterized a family of novel Fe-thiolate compounds with intriguing electronic structures. Theory suggests that $\mathbf{2}$ shows temperature dependent Fe-based spin-crossover which decreases orbital energy separations on the $\mathrm{TTFtt}^{2-}$ core generating significant diradical character. This is corroborated by SXRD data illustrating Fe-based spin-crossover alongside distinctive TTFtt $^{2-}$ bond changes. Mössbauer spectroscopy confirms a spin transition at Fe and the assigned Fe(II) oxidation state while variable temperature electronic spectra and magnetic susceptibility support the proposed change from a closed shell TTFtt ${ }^{2-}$ to a diradical. Finally, the EPR data of $\mathbf{2}$ reveal signals diagnostic of organic diradicals.

Organic diradicals, as seen in $\mathbf{2}$ at low temperature, are relatively rare in general and are unknown in the wellstudied TTF moiety. The reversible modulation of spin between this unusual organic diradical and the Fe centers in 2 represents a fundamentally new form of spin transition and opens the possibility to switch organic diradicals via stimulation of metal-based spin-crossover.

\section{Experimental}

\section{General Synthetic Procedures}

Syntheses and general handling were carried out in a nitrogen-filled MBraun glovebox unless otherwise noted. TTFtt- $\mathrm{C}_{2} \mathrm{H}_{4} \mathrm{CN}$, Fe(TPA)(OTf) $)_{2}(\mathrm{MeCN})_{2}$, dmit-(COPh) and $[\mathrm{Fc}]\left[\mathrm{BAr}_{4}\right]$ were prepared according to literature procedures. ${ }^{59,60,61,62}$ Elemental analyses were performed by Midwest Microlabs. Electrochemical measurements were made on an Epsilon BAS potentiostat. THF and $\mathrm{Et}_{2} \mathrm{O}$ were dried and degassed in a Pure Process Technologies solvent system, stirred over NaK amalgam for $>24$ hours, filtered through alumina, and stored over $4 \AA$ molecular sieves. Methanol was dried with sodium hydroxide overnight, distilled, transferred into the glovebox and stored over $4 \AA$ molecular sieves. 2-Chlorobutane was degassed by the freeze, pump, thaw method and stored over $4 \AA$ molecular sieves. All other solvents were dried and degassed in a Pure Process Technologies solvent system, filtered through activated alumina, and stored over $4 \AA$ molecular sieves. Solvents were tested for $\mathrm{O}_{2}$ and $\mathrm{H}_{2} \mathrm{O}$ with a standard solution of sodium benzophenone ketyl radical. All other reagents were used as purchased without further purification.

\section{Complex Syntheses}

(FeTPA) $)_{2}$ TTFtt (1). TTFtt $\left(\mathrm{C}_{2} \mathrm{H}_{4} \mathrm{CN}\right)_{4} \quad(0.054 \quad \mathrm{~g}, \quad 0.099$ mmol) was stirred with sodium tert-butoxide $0.076 \mathrm{~g}$, $0.791 \mathrm{mmol})$ in THF (6 mL) overnight. The solid material was collected, washed with THF (4 mL x 3), and dried under vacuum to yield a pink powder. The pink solid was then stirred with $\mathrm{Fe}(\mathrm{TPA})(\mathrm{OTf})_{2}(\mathrm{MeCN})_{2}(0.152 \mathrm{~g}, 0.209$ mmol) in THF ( $6 \mathrm{~mL}$ ) for 2 days. The resulting brick red solid was collected, washed with THF ( $4 \mathrm{~mL} \times 3$ ), and dried under vacuum $(0.102 \mathrm{~g}, 100 \%)$. Elemental analysis: expected for $\mathrm{C}_{42} \mathrm{H}_{36} \mathrm{Fe}_{2} \mathrm{~N}_{8} \mathrm{~S}_{8}$ : \% C, 49.41; H, 3.56; N, 10.98 . Found: \% C, 49.78; H, 3.77; N, 10.88.

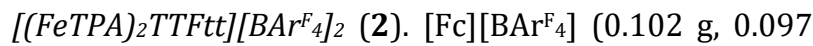
mmol) was dissolved in DCM $(12 \mathrm{~mL})$ and added in 4 portions to (FeTPA)2TTFtt $(0.065 \mathrm{~g}, 0.064 \mathrm{mmol})$ and stirred for 5 minutes after each addition. The mixture was filtered through Celite and the filtrate was layered with petroleum ether. After cooling at $-38{ }^{\circ} \mathrm{C}$ for several days, dark brown crystalline solid formed and was washed with petroleum ether and dried under vacuum (0.081 g, 60\%). Crystals suitable for X-ray diffraction were similarly grown from DCM and petroleum ether. ${ }^{1} \mathrm{H}$ NMR $\left(400 \mathrm{MHz}, 25{ }^{\circ} \mathrm{C}\right.$, $\left.\mathrm{CD}_{2} \mathrm{Cl}_{2}\right) \delta=57.5,56.1,7.7,7.5,-4.8$. Elemental analysis: expected for $\mathrm{C}_{106} \mathrm{H}_{60} \mathrm{~B}_{2} \mathrm{~F}_{48} \mathrm{Fe}_{2} \mathrm{~N}_{8} \mathrm{~S}_{8}$ : \% C, 46.34; $\mathrm{H}, 2.20 ; \mathrm{N}$, 4.08. Found: $\%$ C, 46.54; H, 2.26; N, 4.02. EPR (DCM): $g_{\mathrm{x}}=$ 2.083, $g_{\mathrm{y}}=2.041, g_{\mathrm{z}}=2.047, D=0.028 \mathrm{~cm}^{-1}$. UV-vis-NIR at $20{ }^{\circ} \mathrm{C}$, 2-chlorobutane $\left(\epsilon, \mathrm{M}^{-1} \mathrm{~cm}^{-1}\right): 1025 \mathrm{~nm}\left(8 \times 10^{4}\right), 280$ $\mathrm{nm}\left(3 \times 10^{4}\right)$, $300 \mathrm{~nm}\left(2 \times 10^{4}\right) ;\left(-100^{\circ} \mathrm{C}, 2\right.$-chlorobutane $)$ : $1615 \mathrm{~nm}$ ( $\left.4 \times 10^{4}\right), 325 \mathrm{~nm}\left(3 \times 10^{4}\right), 396 \mathrm{~nm}\left(2 \times 10^{4}\right)$.

$\mathrm{Fe}(\mathrm{TPA})(\mathrm{dmit}) \cdot 0.5 \mathrm{CH}_{2} \mathrm{Cl}_{2}$ (3). The deprotection and metalation procedure was adapted from similar procedures previously reported in the literature ${ }^{63} \mathrm{dmit}-(\mathrm{COPh})(0.080$ g, $0.196 \mathrm{mmol})$ was stirred with sodium methoxide $(0.022$ g, $0.407 \mathrm{mmol})$ in methanol $(6 \mathrm{~mL})$ for 1 hour, then $\mathrm{Fe}(\mathrm{TPA})(\mathrm{OTf})_{2}(\mathrm{MeCN})_{2}(0.152 \mathrm{~g}, 0.209 \mathrm{mmol})$ was added and stirred overnight. Complex 3 was collected, washed with DCM (4 mL x 3), and dried under vacuum to yield a bright red solid $(0.070 \mathrm{~g}, 61 \%)$. Crystals suitable for X-ray diffraction were grown from DCM and petroleum ether. ${ }^{1} \mathrm{H}$ $\operatorname{NMR}\left(400 \mathrm{MHz}, 25{ }^{\circ} \mathrm{C}, \mathrm{CDCl}_{3}\right) \delta=81.1,59.9,59.8,35.7$, -2.99. Elemental analysis: expected for $\mathrm{C}_{21.5} \mathrm{H}_{21} \mathrm{ClFeN}_{4} \mathrm{~S}_{5}$ : \% C, 44.14; H, 3.27; N, 9.58. Found: \% C, 44.65; H, 3.22; N, 9.01. UV-vis-NIR ( $\left.20^{\circ} \mathrm{C}, \mathrm{DCM}\right): 310 \mathrm{~nm}, 498 \mathrm{~nm}$.

$[\mathrm{Fe}(\mathrm{TPA})(\mathrm{dmit})]\left[\mathrm{BAr}_{4}{ }_{4}\right] \quad$ (4). $\mathrm{Fe}(\mathrm{TPA})(\mathrm{dmit}) \cdot 0.5 \mathrm{CH}_{2} \mathrm{Cl}_{2}$ $(0.054 \mathrm{~g}, 0.092 \mathrm{mmol})$ was stirred with $[\mathrm{Fc}]\left[\mathrm{BAr}_{4}{ }^{\mathrm{F}}\right](0.102$ $\mathrm{g}, 0.097 \mathrm{mmol}$ ) in DCM (4 mL) for 10 minutes before filtering through Celite. The filtrate was layered with petroleum ether and cooled to $-38{ }^{\circ} \mathrm{C}$ for several days which yielded green-brown crystals. The solids were washed with petroleum ether and dried under vacuum (0.112 g, 87\%). Crystals which diffracted poorly were similarly grown from DCM and petroleum ether. ${ }^{1} \mathrm{H}$ NMR $\left(400 \mathrm{MHz}, 25{ }^{\circ} \mathrm{C}\right.$, $\left.\mathrm{CD}_{2} \mathrm{Cl}_{2}\right) \delta=16.9,9.1,7.7,7.5$. Elemental analysis: expected for $\mathrm{C}_{47} \mathrm{H}_{32} \mathrm{BF}_{6} \mathrm{FeN}_{4} \mathrm{~S}_{5}$ : \% C, 45.29; $\mathrm{H}, 2.15 ; \mathrm{N}, 3.99$. Found: \% C, 45.33; H, 2.32; N, 3.81. EPR $-g_{\mathrm{x}}=2.056, g_{\mathrm{y}}=2.071, g_{\mathrm{z}}=$ $2.082, \sigma_{\mathrm{gx}}=0.034, \sigma_{\mathrm{gy}}=0.000, \sigma_{\mathrm{gz}}=0.105, \sigma_{\mathrm{B}}=25.994 \mathrm{G}$. UV-vis-NIR at $20^{\circ} \mathrm{C}, \operatorname{DCM}\left(\epsilon, \mathrm{M}^{-1} \mathrm{~cm}^{-1}\right): 417 \mathrm{~nm}\left(2 \times 10^{3}\right)$, $1050 \mathrm{~nm}\left(6 \times 10^{3}\right)$.

\section{Characterization and Analysis Methods}

Magnetometry. Magnetic measurements were carried out on a Quantum Design MPMS3 performed on bulk powder samples in polycarbonate capsules. The powder samples were suspended in an eicosane matrix to prevent movement and protect the sample from incidental air exposure. Frozen solution samples in PEG-2000 were prepared by dissolving 2 and PEG-2000 in DCM, then remov- 
ing DCM under reduced pressure. Diamagnetic corrections for the capsule and eicosane were made by measuring temperature vs. moment in triplicate for each to determine a moment per gram correction. The diamagnetic correction for PEG-2000 was made by measuring sample versus moment to determine a moment per gram correction. Pascal's constants were used to correct for the diamagnetic contribution from the complexes. ${ }^{64}$

Single Crystal X-Ray Diffraction (SXRD). The diffraction data for 2-LT and 2-HT were measured at $100 \mathrm{~K}$ and 298 $\mathrm{K}$, respectively, on a Bruker D8 VENTURE diffractometer equipped with a microfocus Mo-target X-ray tube $(\lambda=$ $0.71073 \AA$ ) and PHOTON 100 CMOS detector. The diffraction data for 3 was measured at $100 \mathrm{~K}$ on a Bruker D8 fixed-chi with PILATUS1M (CdTe) pixel array detector (synchrotron radiation, $\lambda=0.41328 \AA(30 \mathrm{KeV})$ ) at the Chem-MatCARS 15-ID-B beam-line at the Advanced Photon Source (Argonne National Laboratory). Data reduction and integration were performed with the Bruker APEX3 software package (Bruker AXS, version 2015.5-2, 201565). Data were scaled and corrected for absorption effects using the multi-scan procedure as implemented in SADABS (Bruker AXS, version $2014 / 54^{66}$ ). The structures were solved by SHELXT (Version 2014/5567) and refined by a full-matrix least-squares procedure using OLEX26 (XL refinement program version $2018 / 17^{68}$ ). We have only been able to obtain a poor quality structure of $\mathbf{4}$ which verifies its connectivity.

X-Ray Powder Diffraction (XRPD) Diffraction patterns were collected on a SAXSLAB Ganesha diffractometer with a Cu K- $\alpha$ source $(\lambda=1.54 \AA)$ in wide angle X-ray scattering (WAXS) transmission mode. The samples were contained in a $\sim 1 \mathrm{~mm}$ diameter borosilicate capillary tube. A correction was made to subtract the broad peak from the capillary around $16-25^{\circ}(2 \theta)$ from the baseline.

$U V$-vis-NIR. Variable temperature UV-vis-NIR measurements were performed on a Shimadzu UV-3600 Plus dual beam spectrophotometer with a Unisoku CoolSpeK 203-B cryostat. UV-vis region spectra were collected on Thermo Scientific Evolution 300 spectrometer with the VISIONpro software suite. Samples were stirred during cooling and during measurements colder than room temperature. Background spectra of the cuvette and solvent were collected at maximum and minimum temperatures within the range to account for temperature dependence of the background.

Cyclic Voltammetry. Electrochemical measurements were performed using a BAS Epsilon potentiostat and analyzed using BAS Epsilon software version 1.40.67NT. Cyclic voltammetry measurements were made using a glassy carbon working electrode, platinum wire counter electrode, and silver wire pseudo-reference electrode, and referenced to internal $\mathrm{Fc} / \mathrm{Fc}^{+}$.

EPR Spectroscopy. EPR spectra were recorded on a Bruker Elexsys E500 spectrometer equipped with an Oxford ESR 900 X-band cryostat and a Bruker Cold-Edge Stinger. Simulation of EPR spectra was performed using a least-squares fitting method with the SpinCount program.

Mössbauer Spectroscopy. Zero-field iron-57 Mössbauer spectra were with a constant acceleration spectrometer and a rhodium embedded cobalt-57 source. Prior to measurements, the spectrometer was calibrated at $295 \mathrm{~K}$ with $\alpha$-iron foil. Samples were prepared in a $\mathrm{N}_{2}$-filled glovebox where powdered samples were placed in a polyethylene cup and frozen in liquid nitrogen prior to handling in air. All spectra were analyzed using the WMOSS Mössbauer Spectral Analysis Software. ${ }^{69}$

FT-IR Spectroscopy. Powder samples for FT-IR were formed into pellets in a potassium bromide matrix. Spectra were acquired in absorbance mode on a Bruker Tensor II with background subtractions used to account for air. A linear baseline correction was applied.

NMR Spectroscopy. ${ }^{1} \mathrm{H}$ and ${ }^{2} \mathrm{H}$ spectra were recorded on Bruker DRX 400 or 500 spectrometers. Chemical shifts of ${ }^{1} \mathrm{H}$ NMR are reported in ppm units referenced to residual solvent resonances. Variable temperature ${ }^{2} \mathrm{H}$ NMR spectra were collected in $95 / 5 \mathrm{w} / \mathrm{w} \%$ of 2 -chlorobutane $/ \mathrm{C}_{6} \mathrm{D}_{6}$ with a capillary of $90 / 10 \mathrm{w} / \mathrm{w} \%$ 2-chlorobutane $/ \mathrm{C}_{6} \mathrm{D}_{6}$. The temperature dependent change in density of both 2chlorobutane and $\mathrm{C}_{6} \mathrm{D}_{6}$ was accounted for at each temperature point, to yield an accurate solution concentration. Pascal's constants were used to correct for the diamagnetic contribution of the complex.

Fitting of Magnetic and NIR Data of 2 to a Boltzmann Distribution. The magnetic data and NIR spectroscopic data of $\mathbf{2}$ were fit to a Boltzmann distribution of the form:

$$
\frac{p_{\mathrm{HT}}}{p_{\mathrm{LT}}}=e^{\Delta H / k_{B}\left(T-T_{1 / 2}\right)}
$$

Where $p_{\text {HT }}$ and $p_{\text {LT }}$ are the fractions of HT and LT forms of $\mathbf{2}$, respectively, $\Delta H$ is the energy difference between $\mathbf{2 - H T}$ and 2-LT, $k_{\mathrm{B}}$ is the Boltzmann constant, $T$ is the temperature of the measurement, and $T_{1 / 2}$ is the transition temperature.

For the spectroscopic data, the fraction of 2-HT was estimated by assuming the fraction of 2 -HT at $173 \mathrm{~K}$ is zero, due to the lack of the characteristic NIR signal around $1100 \mathrm{~nm}$. The fraction of $\mathbf{2}$ - $\mathbf{L T}$ at $173 \mathrm{~K}$ is correspondingly $100 \%$, and a linear relationship between intensity and concentration of 2-LT was assumed in order to calculate the fraction of each form at various temperatures.

TD-DFT Calculations. TD-DFT calculations were performed with ORCA $^{70,71,72,73}$ software suite using timedependent density functional theory (TD-DFT). The PBE0 functional was used with a basis set of def2-TZVPP on Fe and def2-TZVP on all other atoms. Furthermore, an effective core potential of SDD was used on Fe. Starting coordinates for all calculations were from crystal structures determined by single crystal X-ray diffraction at $100 \mathrm{~K}$ or 293 $\mathrm{K}$ as indicated. Simulations of UV-vis spectra were generated using the orca_mapspc function with line broadening of $2000 \mathrm{~cm}^{-1}$. Molecular orbitals were generated using the orca_plot function and visualized in Avogadro with an iso value of 0.3 .

To calibrate the calculations, the electronic spectrum of the previously reported ${ }^{34}$ reference complex [(dppeNi $)_{2} \mathrm{TTFtt}_{[}\left[\mathrm{BAr}_{4}\right]_{2}$ - which features a doubly oxidized TTF core, as in $\mathbf{2}$ - was calculated by the same methods using the reported crystal structure. Using the experimental spectral data, a weighted calibration was calculated using the shifts to the primary absorbances (i.e. 
at 1039 and $516 \mathrm{~nm}$ ). This calibration was then applied to the calculated spectra of 2 . The predicted spectra of both $S$ $=0$ 2-LT and $S=42$-HT match well to the spectra of 2 at $20{ }^{\circ} \mathrm{C}$. Conversely, the low temperature spectrum of 2 only matches well with the predicted spectrum of the $S=1$ spin state of the 2-LT structure. The are many predicted transitions for the $S=4$ state of $\mathbf{2 - H T}$ and their orbital composition is complicated and highly mixed. Nevertheless, the predicted spectrum matches experiment well, and TTFtt character is apparent in the NIR transitions. The predicted transitions of the closed shell $S=0$ state of 2-LT are much simpler, match those in $\left[(\mathrm{dppeNi})_{2} \mathrm{TTFtt}_{[}\left[\mathrm{BAr}^{\mathrm{F}}\right]_{2}\right.$, and provide a clearer interpretation of the orbital composition. While we don't believe that the closed shell $S=0$ state of 2LT is an accurate electronic structure description, it does serve as a simpler model to understand the orbital contributions of the NIR features of $\mathbf{2}$ at high temperature.

V2RDM and DFT Methods. Variational 2-electron reduced density matrix (V2RDM) and DFT calculations were carried out to elucidate the electronic structure of 2 . The V2RDM method allows large complete active space selfconsistent field (CASSCF) to be carried out with polynomial $\mathrm{O}\left(\mathrm{r}^{6}\right)$ computational scaling, enabling calculations to be carried out that remain out of reach of traditional wave function based CASSCF methods which scale exponentially. ${ }^{74}$ This is achieved by formulating the system energy as a linear functional of the 2RDM:

$$
\mathrm{E}=\operatorname{Tr}\left[{ }^{2} \mathrm{~K}^{2} \mathrm{D}\right]
$$

where ${ }^{2} \mathrm{~K}$ is the 2-electron reduced Hamiltonian, and ${ }^{2} \mathrm{D}$ is the 2-RDM. Minimization of the energy is subject to a set of constraints on the 2RDM that are termed $\mathrm{N}$ representability constraints and ensure that variationally obtained 2RDM corresponds to a physically feasible system..$^{75,76}$

$$
\begin{aligned}
{ }^{2} \mathrm{D} & \geq 0 \\
{ }^{2} \mathrm{Q} & \geq 0 \\
{ }^{2} \mathrm{G} & \geq 0
\end{aligned}
$$

This procedure is carried out using a semi-definite program. ${ }^{55,56}$ The V2RDM method has been demonstrated to recover the vast majority of the correlation energy in strongly correlated systems and has recently been applied to a range of transition metal systems to successfully explain their electronic structure. ${ }^{77,78,34}$ To ensure that the obtained NO and spin state splitting picture of the LT form of $\mathbf{2}$ is not an artifact of the choice of active space orbitals and size or basis set further calculations on the singlet and triplet state were carried out using a $[16,14]$ active apace and 6-31G basis sets and a $[14,14]$ active space with a larger 6-31G* basis set, each time forcing iron $3 \mathrm{~d}$ orbitals into the active space for the initial guess. The data are shown in Table S1. While the $[16,14]$ active space is not large enough to account for the full correlation energy yielding a significantly smaller singlet-triplet gap of $\Delta \mathrm{E}(\mathrm{T}-\mathrm{S})=210$ $\mathrm{cm}^{-1}$, the $[14,14] / 6-31 \mathrm{G}^{*}$ calculation gives an almost identical gap to the larger $[18,20]$ calculation with $\Delta \mathrm{E}(\mathrm{T}-\mathrm{S})=$ $379 \mathrm{~cm}^{-1}$. In both cases the CASSCF routine rotates the NOs with iron $3 \mathrm{~d}$ contributions back into the core and virtual orbitals, obtaining identical Nos as those obtained in the $[18,20]$ calculation.

\section{ASSOCIATED CONTENT}

Additional supporting data and experimental methods are available in the Supplementary Information file.

\section{AUTHOR INFORMATION}

\section{Corresponding Author \\ *Email: jsanderson@uchicago.edu}

\section{Author Contributions}

A.K. synthesized compounds 1-4, TPA and Fe(OTf) $)_{2}(\mathrm{MeCN})_{2}$ starting materials, collected and analyzed UV-Vis-NIR, magnetic, ${ }^{1} \mathrm{H}$ NMR, and SXRD data for 2, performed data work up for Mössbauer and ${ }^{2} \mathrm{H}$ NMR, and contributed to data work up of EPR and TD-DFT. J.X. synthesized TTFtt- $\mathrm{C}_{2} \mathrm{H}_{4} \mathrm{CN}$ and dmit ${ }^{2-}$ proligands and $\mathrm{NaBArF}_{4}$. J.-N.B. performed DFT and V2RDM computational calculations. K.A.J. performed TD-DFT. A.J.M. collected SXRD data for $\mathbf{3}$ and performed data work up for all SXRD. E.A.H. collected and simulated EPR data. K.A.C. and J.A.V.-M. collected Mössbauer data. A.S.F. assisted in SXRD data workup. J.W. Kurutz collected ${ }^{2}$ H NMR. D.A.M. and J.S.A. contributed intellectually and supervised the project. A.K., J.X., and J.-N.B. contributed intellectually. A.K. and J.-N.B. wrote the manuscript. All authors have given approval to the final version of the manuscript. $\$$ These authors contributed equally.

\section{ACKNOWLEDGMENT}

Parts of this work were carried out at the Soft Matter Characterization Facility at the Pritzker School of Molecular Engineering at the University of Chicago. J. S. A. and D. A. M. gratefully acknowledge support for this work from the U. S. Department of Energy, Office of Science, Office of Basic Energy Sciences, under Award No. DE-SC0019215. J. S. A. also gratefully acknowledges support from 3M corporation through an NTFA and the Army Research Office under Grant Number W911NF-20-1-0091. K.A.C. acknowledges support from the NSF GRFP through DGE-11842165. This work was supported by the Chicago MRSEC, which is funded by NSF through Grant DMR-1420709. The University of Chicago is thanked for startup funds. Use of the Advanced Photon Source at Argonne National Laboratory was supported by the U. S. Department of Energy, Office of Science, Office of Basic Energy Sciences, under Contract No. DE-AC02-06CH11357, and we thank Dr. YuSheng Chen and Dr. Suyin Grass Wang for assistance with SXRD data acquisition at beamline 15-ID-B, C, and D. We also thank professors Dave Harris, Danna Freedman, and Jeremy Smith for useful discussions and assistance with Mössbauer spectroscopy.

\section{REFERENCES}

(1) Abe, M., Diradicals. Chem. Rev. 2013, 113 (9), 7011-7088.

(2) Morita, Y.; Suzuki, S.; Sato, K.; Takui, T., Synthetic organic spin chemistry for structurally well-defined open-shell graphene fragments. Nat. Chem. 2011, 3 (3), 197-204.

(3) Kamada, K.; Ohta, K.; Shimizu, A.; Kubo, T.; Kishi, R.; Takahashi, H.; Botek, E.; Champagne, B.; Nakano, M., Singlet Diradical Character from Experiment. J. Phys. Chem. Lett. 2010, 1 (6), 937-940.

(4) Smith, M. B.; Michl, J., Singlet Fission. Chem. Rev. 2010, 110 (11), 6891-6936.

(5) Schweicher, G.; Garbay, G.; Jouclas, R.; Vibert, F.; Devaux, F.; Geerts, Y. H., Molecular Semiconductors for Logic Operations: Dead-End or Bright Future? Adv. Mater. 2020, 32 (10), 1905909.

(6) Gallagher, N.; Zhang, H.; Junghoefer, T.; Giangrisostomi, E.; Ovsyannikov, R.; Pink, M.; Rajca, S.; Casu, M. B.; Rajca, A., Thermal- 
ly and Magnetically Robust Triplet Ground State Diradical. J. Am. Chem. Soc. 2019, 141 (11), 4764-4774.

(7) Tang, S.; Zhang, L.; Ruan, H.; Zhao, Y.; Wang, X., A Magnetically Robust Triplet Ground State Sulfur-Hydrocarbon Diradical Dication. J. Am. Chem. Soc. 2020.

(8) Fukuzumi, S.; Ohkubo, K.; Ishida, M.; Preihs, C.; Chen, B.; Borden, W. T.; Kim, D.; Sessler, J. L., Formation of Ground State Triplet Diradicals from Annulated Rosarin Derivatives by Triprotonation. J. Am. Chem. Soc. 2015, 137 (31), 9780-9783.

(9) Suzuki, S.; Furui, T.; Kuratsu, M.; Kozaki, M.; Shiomi, D.; Sato, K.; Takui, T.; Okada, K., Nitroxide-Substituted Nitronyl Nitroxide and Iminonitroxide. J. Am. Chem. Soc. 2010, 132 (45), 1590815910.

(10) Su, Y.; Wang, X.; Wang, L.; Zhang, Z.; Wang, X.; Song, Y.; Power, P. P., Thermally controlling the singlet-triplet energy gap of a diradical in the solid state. Chem. Sci. 2016, 7 (10), 6514-6518.

(11) Wang, J.; Hou, L.; Browne, W. R.; Feringa, B. L., Photoswitchable Intramolecular Through-Space Magnetic Interaction. J. Am. Chem. Soc. 2011, 133 (21), 8162-8164.

(12) Zhang, R.; Peterson, J. P.; Fischer, L. J.; Ellern, A.; Winter, A. H., Effect of Structure on the Spin-Spin Interactions of Tethered Dicyanomethyl Diradicals. J. Am. Chem. Soc. 2018, 140 (43), 1430814313..

(13) Trabolsi, A.; Khashab, N.; Fahrenbach, A. C.; Friedman, D. C.; Colvin, M. T.; Cotí, K. K.; Benítez, D.; Tkatchouk, E.; Olsen, J.-C.; Belowich, M. E.; Carmielli, R.; Khatib, H. A.; Goddard, W. A.; Wasielewski, M. R.; Stoddart, J. F., Radically enhanced molecular recognition. Nature Chem. 2010, 2 (1), 42-49.

(14) Schuler, B.; Fatayer, S.; Mohn, F.; Moll, N.; Pavliček, N.; Meyer, G.; Peña, D.; Gross, L., Reversible Bergman cyclization by atomic manipulation. Nature Chem. 2016, 8 (3), 220-224.

(15) Yin, X.; Low, J. Z.; Fallon, K. J.; Paley, D. W.; Campos, L. M., The butterfly effect in bisfluorenylidene-based dihydroacenes: aggregation induced emission and spin switching. Chemical Science 2019, 10 (46), 10733-10739.

(16) Tissot, A.; Shepherd, H. J.; Toupet, L.; Collet, E.; Sainton, J.; Molnár, G.; Guionneau, P.; Boillot, M.-L., Temperature- and Pressure-Induced Switching of the Molecular Spin State of an Orthorhombic Iron(III) Spin-Crossover Salt. European Journal of Inorg. Chem. 2013, 2013 (5-6), 1001-1008.

(17) García-López, V.; Waerenborgh, J. C.; Vieira, B. J. C.; ClementeLeón, M.; Coronado, E., Iron(II) complexes of tris(2pyridylmethyl)amine (TPMA) and neutral bidentate ligands showing thermal- and photo-induced spin-crossover. Dalton Trans. 2018, 47 (27), 9156-9163.

(18) Shatruk, M.; Phan, H.; Chrisostomo, B. A.; Suleimenova, A., Symmetry-breaking structural phase transitions in spin-crossover complexes. Coord. Chem. Rev. 2015, 289-290, 62-73.

(19) Mathonière, C.; Lin, H.-J.; Siretanu, D.; Clérac, R.; Smith, J. M. "Photoinduced Single-Molecule Magnet Properties in a FourCoordinate Iron(II) Spin Crossover Complex" J. Am. Chem. Soc. 2013, 135, 19083-19086.

(20) Feng, X.; Mathonière, C.; Jeon, I.-R.; Rouzières, M.; Ozarowski, A.; Aubrey, M. L.; Gonzalez, M. I.; Clérac, R.; Long, J. R., Tristability in a Light-Actuated Single-Molecule Magnet.J. Am. Chem. Soc. 2013, 135 (42), 15880-15884.

(21) Arroyave, A.; Lennartson, A.; Dragulescu-Andrasi, A.; Pedersen, K. S.; Piligkos, S.; Stoian, S. A.; Greer, S. M.; Pak, C.; Hietsoi, O.; Phan, H.; Hill, S.; McKenzie, C. J.; Shatruk, M., Spin-crossover in $\mathrm{Fe}(\mathrm{II}) \quad$ Complexes with $\mathrm{N}_{4} \mathrm{~S}_{2}$ Coordination. Inorg. Chem. 2016, 55 (12), 5904-5913.

(22) Senthil Kumar, K.; Ruben, M., Emerging trends in spincrossover (SCO) based functional materials and devices. Coord. Chem. Rev. 2017, 346, 176-205.

(23) Rose, B. D.; Sumner, N. J.; Filatov, A. S.; Peters, S. J.; Zakharov, L. N.; Petrukhina, M. A.; Haley, M. M., Experimental and Computational Studies of the Neutral and Reduced States of Indeno[1,2b]fluorene. J. Am. Chem. Soc. 2014, 136 (25), 9181-9189.
(24) Thorarinsdottir, A. E.; Harris, T. D., Metal-Organic Framework Magnets. Chem. Rev. 2020.

(25) Alexandropoulos, D. I.; Dolinar, B. S.; Vignesh, K. R.; Dunbar, K. R., Putting a New Spin on Supramolecular Metallacycles: $\mathrm{Co}_{3}$ Triangle and $\mathrm{Co}_{4}$ Square Bearing Tetrazine-Based Radicals as Bridges. J. Am. Chem. Soc. 2017, 139 (32), 11040-11043.

(26) Ylijoki, K. E. O.; Lavy, S.; Fretzen, A.; Kündig, E. P.; Berclaz, T.; Bernardinelli, G.; Besnard, C., A Synthetic and Mechanistic Investigation of the Chromium Tricarbonyl-Mediated MasamuneBergman Cyclization. Direct Observation of a Ground-State Triplet p-Benzyne Biradical. Organometallics 2012, 31 (15), 5396-5404.

(27) O'Connor, J. M.; Friese, S. J., Transition-Metal Hydrides as Hydrogen Atom Donors: Stronger Metal-Hydrogen Bonds Can Be Advantageous. Organometallics 2008, 27 (17), 4280-4281..

(28) Barclay, T. M.; Hicks, R. G.; Lemaire, M. T.; Thompson, L. K., Weak Magnetic Coupling of Coordinated Verdazyl Radicals through Diamagnetic Metal Ions. Synthesis, Structure, and Magnetism of a Homoleptic Copper(I) Complex. Inorg. Chem. 2001, 40 (25), 6521-6524.

(29) Gardiner, M. G.; Hanson, G. R.; Henderson, M. J.; Lee, F. C.; Raston, C. L., Paramagnetic Bis(1,4-di-tert-butyl-1,4diazabutadiene) Adducts of Lithium, Magnesium, and Zinc. Inorg. Chem. 1994, 33 (11), 2456-2461.

(30) Paul, N. D.; Rana, U.; Goswami, S.; Mondal, T. K.; Goswami, S., Azo Anion Radical Complex of Rhodium as a Molecular Memory Switching Device: Isolation, Characterization, and Evaluation of Current-Voltage Characteristics. J. Am. Chem. Soc. 2012, 134 (15), 6520-6523.

(31) Petrenko, T.; Ray, K.; Wieghardt, K. E.; Neese, F., Vibrational Markers for the Open-Shell Character of Transition Metal Bisdithiolenes: An Infrared, Resonance Raman, and Quantum Chemical Study. J. Am. Chem. Soc. 2006, 128 (13), 4422-4436.

(32) Wang, H.-Y.; Cui, L.; Xie, J.-Z.; Leong, C. F.; D’Alessandro, D. M.; Zuo, J.-L., Functional coordination polymers based on redox-active tetrathiafulvalene and its derivatives. Coord. Chem. Rev. 2017, 345, 342-361.

(33) Segura, J. L.; Martin, N., New concepts in tetrathiafulvalene chemistry. Angew. Chem., Int. Ed. 2001, 40 (8), 1372-1409.

(34) Xie, J.; Boyn, J.-N.; Filatov, A. S.; McNeece, A. J.; Mazziotti, D. A.; Anderson, J. S., Redox, transmetalation, and stacking properties of tetrathiafulvalene-2,3,6,7-tetrathiolate bridged tin, nickel, and palladium compounds. Chem. Sci. 2020.

(35) Gao, F.; Zhu, F.-F.; Wang, X.-Y.; Xu, Y.; Wang, X.-P.; Zuo, J.-L., Stabilizing Radical Cation and Dication of a Tetrathiafulvalene Derivative by a Weakly Coordinating Anion. Inorg. Chem. 2014, 53 (10), 5321-5327.

(36) Hilfiger, M. G.; Chen, M.; Brinzari, T. V.; Nocera, T. M.; Shatruk, M.; Petasis, D. T.; Musfeldt, J. L.; Achim, C.; Dunbar, K. R. An Unprecedented Charge Transfer Induced Spin Transition in an $\mathrm{Fe}-\mathrm{Os}$ Cluster Angew. Chem. Int. Ed. 2010, 49, 1410-1413.

(37) Min, K. S.; Swierczek, K.; DiPasquale, A. G.; Rheingold, A. L.; Reiff, W. M.; Arif, A. M.; Miller, J. S., A dinuclear iron(II) complex, $\left[(\mathrm{TPyA}) \mathrm{Fe}^{\mathrm{II}}\left(\mathrm{THBQ}_{2}-\right) \mathrm{Fe}^{\mathrm{II}}(\mathrm{TPyA})\right]\left(\mathrm{BF}_{4}\right)_{2} \quad[\mathrm{TPyA}=\operatorname{tris}(2-$ pyridylmethyl)amine; $\mathrm{THBQ}^{2-}=2,3,5,6$-tetrahydroxy-1,4benzoquinonate] exhibiting both spin-crossover with hysteresis and ferromagnetic exchange. Chem. Commun., 2008, (3), 317-319. (38) Zheng, C.; Xu, J.; Wang, F.; Tao, J.; Li, D., Spin-crossover and reversible single-crystal to single-crystal transformation behaviour in two cyanide-bridged mixed-valence $\left\{\mathrm{Fe}^{\mathrm{III}_{2}} \mathrm{Fe}^{\mathrm{II}_{2}}\right\}$ clusters. Dalton Trans. 2016, 45 (43), 17254-17263.

(39) Paulsen, H.; Grünsteudel, H.; Meyer-Klaucke, W.; Gerdan, M.; Grünsteudel, H. F.; Chumakov, A. I.; Rüffer, R.; Winkler, H.; Toftlund, H.; Trautwein, A. X. The spin-crossover complex [Fe(tpa)(NCS) 2 ]. Eur. Phys. J. B, 2001, 23, 463-472.

(40) Polam, J. R.; Wright, J. L.; Christensen, K. A.; Walker, F. A.; Flint, H.; Winkler, H.; Grodzicki, M.; Trautwein, A. X., Valence Electron Cloud Asymmetry from Two Points of View: A Correlation between Mössbauer Quadrupole Splittings and ${ }^{57} \mathrm{Fe}$ NMR Chemi- 
cal Shifts of Diamagnetic Iron(II) Porphyrinates. J. Am. Chem. Soc. 1996, 118 (22), 5272-5276.

(41) Min, K. S.; DiPasquale, A.; Rheingold, A. L.; Miller, J. S., RoomTemperature Spin-crossover Observed for $\left[\left(\mathrm{TPyA}^{2} \mathrm{Fe}^{\mathrm{II}}\left(\mathrm{DBQ}^{2-}\right) \mathrm{FeII}(\mathrm{TPyA})\right]^{2+} \quad[\right.$ TPyA $=$ Tris(2pyridylmethyl)amine; $\mathrm{DBQ}^{2-}=2,5$-Di-tert-butyl-3,6-dihydroxy1,4-benzoquinonate]. Inorg. Chem. 2007, 46 (4), 1048-1050.

(42) Nihei, M.; Shiga, T.; Maeda, Y.; Oshio, H., Spin-crossover iron(III) complexes. Coord. Chem. Rev. 2007, 251 (21), 2606-2621.

(43) Olguín, J.; Brooker, S., Spin-crossover active iron(II) complexes of selected pyrazole-pyridine/pyrazine ligands. Coord. Chem. Rev. 2011, 255 (1), 203-240.

(44) Scepaniak, J. J.; Harris, T. D.; Vogel, C. S.; Sutter, J.; Meyer, K.; Smith, J. M., Spin-crossover in a Four-Coordinate Iron(II) Complex. J. Am. Chem. Soc. 2011, 133 (11), 3824-3827.

(45) Bellec, N.; Vacher, A.; Barrière, F.; Xu, Z.; Roisnel, T.; Lorcy, D., Interplay between Organic-Organometallic Electrophores within Bis(cyclopentadienyl)Molybdenum Dithiolene Tetrathiafulvalene Complexes. Inorg. Chem. 2015, 54 (10), 5013-5020.

(46) Xu, C.-H.; Sun, W.; Zhang, C.; Zhou, C.; Fang, C.-J.; Yan, C.-H., Luminescence Switching of a Cyclometalated Iridium(III) Complex through a Redox-active Tetrathiafulvalene-based Ligand. Chem. Eur. J. 2009, 15 (35), 8717-8721.

(47) Matsubayashi, G.-e.; Takahashi, K.; Tanaka, T., X-Ray crystal structure of bis(N-ethylpyridinum) bis[4,5-dimercapto-1,3dithiole-2-thionato(2-)]cuprate(II) and electrical properties of its oxidized salts. Dalton Trans. 1988, (4), 967-972.

(48) Ferreira, G. B.; Hollauer, E.; Comerlato, N. M.; Wardell, J. L., An experimental and theoretical study of the electronic spectra of tetraethylammonium [bis(1,3-dithiole-2-thione-4,5dithiolato)zincate(II)], [NEt $]_{2}\left[\mathrm{Zn}(\mathrm{dmit})_{2}\right]$, and tetraethylammonium [bis(1,3-dithiole-2-one-4,5-dithiolato)zincate(II)], $\left[\mathrm{NEt}_{4}\right]_{2}\left[\mathrm{Zn}(\mathrm{dmio})_{2}\right]$. Inorganica Chim. Acta 2006, 359 (4), 12391247.

(49) Hitomi, Y.; Furukawa, S.; Higuchi, M.; Shishido, T.; Tanaka, T., Alkane hydroxylation catalyzed by a series of mononuclear nonheme iron complexes containing 4-nitropyridine ligands. J. Mol. Catal. A: Chem. 2008, 288 (1), 83-86.

(50) Kawabata, K.; Nakano, M.; Tamura, H.; Matsubayashi, G.-E., Crystal structures of $\left[\mathrm{Rh}\left(\eta^{5}-\mathrm{C}_{5} \mathrm{H}_{5}\right)\left(\mathrm{C}_{3} \mathrm{~S}_{5}\right)\right]$ and $\left[\mathrm{Rh}\left(\eta^{5-}\right.\right.$ $\left.\left.\mathrm{C}_{5} \mathrm{Me}_{5}\right)\left(\mathrm{C}_{3} \mathrm{~S}_{5}\right)\right]_{2}$ and properties of their oxidized species. J. Organomet. Chem. 2004, 689 (2), 405-410.

(51) Akasaka, T.; Nakano, M.; Tamura, H.; Matsubayashi, G.-E., Preparation and Properties of Tin(IV) Complexes with the SulfurRich Dithiolate $\mathrm{C}_{3} \mathrm{~S}_{5}$ and $\mathrm{C}_{8} \mathrm{H}_{4} \mathrm{~S}_{8}$ Ligands and Their Oxidation. Bull. Chem. Soc. Jpn. 2002, 75 (12), 2621-2628.

(52) Becke, A. D, Density-functional thermochemistry. III. the role of exact exchange. J. Chem. Phys., 1993, 98 (7), 5648-5652.

(53) Krishnan, R.; Binkley, J. S.; Seeger, R.; Pople, J. A., Selfconsistent molecular orbital methods. XX. A basis set for correlated wave functions. J. Chem. Phys. 1980, 72 (1), 650-654.

(54) Frisch, M. J. et al., Gaussian 16 Revision A.01, 2016. Gaussian Inc. Wallingford CT.

(55) Mazziotti, D. A. Large-Scale Semidefinite Programming for Many-Electron Quantum Mechanics Phys. Rev. Lett. 2011, 106 (8), 083001.

(56) Mazziotti, D. A. Realization of quantum chemistry without wave functions through first-order semidefinite programming. Phys. Rev. Lett., 2004, 93 (21), 213001.

(57) Schlimgen, A. W.; Heaps, C. W.; Mazziotti, D. A., Entangled Electrons Foil Synthesis of Elusive Low-Valent Vanadium Oxo Complex J. Phys. Chem. Lett. 2016, 7 (4), 627-631.

(58) Maple Quantum Chemistry Package from RDMChem 2020. Maplesoft, a division of Waterloo Maple Inc., Waterloo, Ontario.

(59) Hansen, T. K., et al., 4,5-DIBENZOYL-1,3-DITHIOLE-1THIONE, Org. Synth. 1996, 73, 270.

(60) Svenstrup, N.; Rasmussen, K. M.; Hansen, T. K. \& Becher, J. The Chemistry of TTFTT; 1: New Efficient Synthesis and Reactions of Tetrathiafulvalene-2,3,6,7-Tetrathiolate (TTFTT): An Im- portant Building Block in TTF-Syntheses. Synthesis. 1994, 8, 809812.

(61) Chávez, I.; Alvarez-Carena, A.; Molins*, E.; Roig, A.; Maniukiewicz, W.; Arancibia, A.; Arancibia, V.; Brand, H.; Manuel Manríquez*, J., Selective Oxidants for Organometallic Compounds Containing a Stabilising Anion of Highly Reactive Cations: $\left.\left(3,5\left(\mathrm{CF}_{3}\right)_{2} \mathrm{C}_{6} \mathrm{H}_{3}\right)_{4} \mathrm{~B}^{-}\right) \mathrm{Cp}_{2} \mathrm{Fe}^{+}$and $\left.\left(3,5\left(\mathrm{CF}_{3}\right)_{2} \mathrm{C}_{6} \mathrm{H}_{3}\right)_{4} \mathrm{~B}^{-}\right) \mathrm{Cp}^{*} 2 \mathrm{Fe}^{+}$. J. Organomet. Chem. 2000, 601 (1), 126-132.

(62) Diebold, A.; Hagen, K. S., Iron(II) Polyamine Chemistry: Variation of Spin State and Coordination Number in Solid State and Solution with Iron(II) Tris(2-pyridylmethyl)amine Complexes. Inorg. Chem. 1998, 37 (2), 215-223.

(63) Zadrozny, J. M.; Greer, S. M.; Hill, S.; Freedman, D. E., A flexible iron(II) complex in which zero-field splitting is resistant to structural variation. Chem. Sci. 2016, 7 (1), 416-423.

(64) Bain, G. A.; Berry, J. F., Diamagnetic Corrections and Pascal's Constants. J. Chem. Ed. 2008, 85 (4), 532.

(65) Krause, L.; Herbst-Irmer, R.; Sheldrick, G. M.; Stalke, D. Comparison of Silver and Molybdenum Microfocus X-Ray Sources for Single-Crystal Structure Determination J. Appl. Crystallogr. 2015, $48,3-10$.

(66) Sheldrick, G.M. Shelxt - Integrated Space-Group and CrystalStructure Determination Acta Crystallogr., Sect. A: Found. Adv. 2015, 71, 3-8

(67) Dolomanov, O. V.; Bourhis, L. J.; Gildea, R. J.; Howard, J. A. K.; Puschmann, H. "Olex2: A Complete Structure Solution, Refinement and Analysis Program" J. Appl. Crystallogr. 2009, 42, 339-341.

(68) Sheldrick, G.M. Crystal Structure Refinement with ShelXL Crystal Acta Crystallogr., Sect. C: Struct. Chem. 2015, 71, 3-8.

(69) Prisecaru, I. "WMOSS4 Mössbauer Spectral Analysis Software, www.wmoss.org, 2009-2016"

(70) Neese, F. "The Orca Program System" Wiley Interdisciplinary Reviews: Computational Molecular Science, 2, 73-78, doi: 10.1002/wcms.81 (2012)

(71) H - Kr: A. Schaefer, H. Horn and R. Ahlrichs, Fully optimized contracted Gaussian basis sets for atoms Li to Kr J. Chem. Phys. 1992, 97, 2571.

(72) Rb - Xe: A. Schaefer, C. Huber and R. Ahlrichs, Fully optimized contracted Gaussian basis sets of triple zeta valence quality for atoms Li to Kr J. Chem. Phys. 1994, 100, 5829.

(73) F. Weigend, R. Ahlrichs, Balanced basis sets of split valence, triple zeta valence and quadruple zeta valence quality for $\mathrm{H}$ to $\mathrm{Rn}$ : Design and assessment of accuracy Phys. Chem. Chem. Phys. 2005, 7, 3297.

(74) Mazziotti, D. A. Two-Electron Reduced Density Matrix as the Basic Variable in Many-Electron Quantum Chemistry and Physics. Chem. Rev. 2012, 112, 244-262.

(75) Mazziotti, D. A. Variational minimization of atomic and molecular ground-state energies via the two-particle reduced density matrix. Phys. Rev. A, 2002, 65, 062511

(76) Mazziotti, D. A. Structure of fermionic density matrices: Complete n-representability conditions. Phys. Rev. Lett., 2012, 108, 263002.

(77) McIsaac, A.R. \& Mazziotti, D. A. Ligand non-innocence and strong correlation in manganese superoxide dismutase mimics. Phys. Chem. Chem. Phys., 2017, 19, 4656-4660.

(78) Montgomery, J.M. \& Mazziotti, D. A. Strong electron correlation in nitrogenase cofactor, FeMoco. J. Phys. Chem. A, 2018, 122(22), 4988-4996. 
Insert Table of Contents artwork here
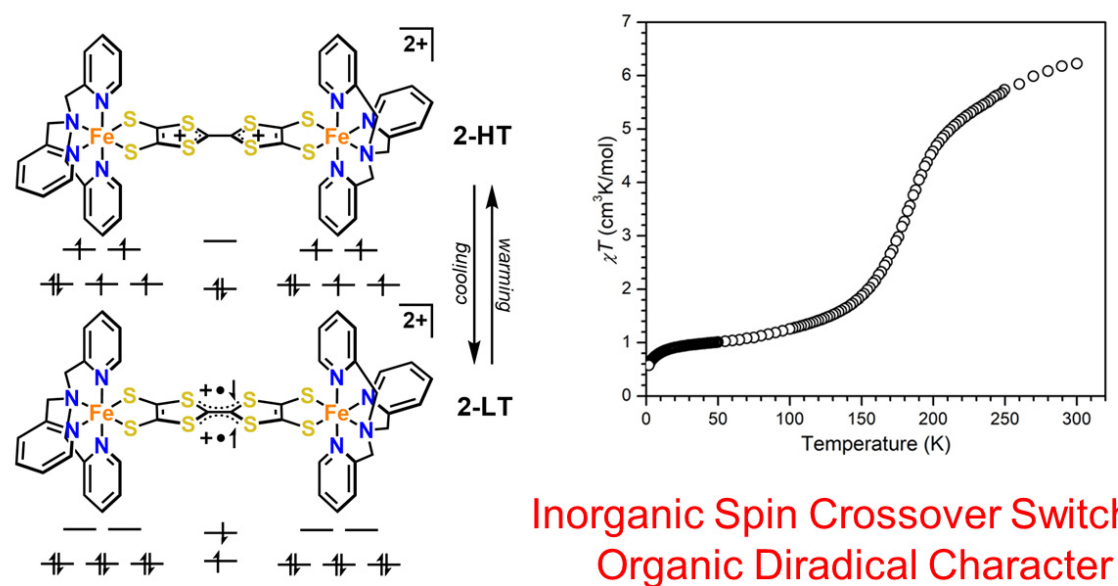

Inorganic Spin Crossover Switches

Organic Diradical Character 